\title{
RESEARCH
}

Open Access

\section{Elamipretide (SS-31) improves mitochondrial dysfunction, synaptic and memory impairment induced by lipopolysaccharide in mice}

Weixing Zhao', Zhipeng Xu' ${ }^{1}$ Jiangbei Cao ${ }^{1}$, Qiang Fu', Yishuang Wu', Xiaoying Zhang ${ }^{1}$, Yue Long ${ }^{1}$, Xuan Zhang ${ }^{1}$, Yitian Yang ${ }^{1}$, Yunfeng $\mathrm{Li}^{2}$ and Weidong $\mathrm{Mi}^{1 *}$

\begin{abstract}
Background: It is widely accepted that mitochondria have a direct impact on neuronal function and survival. Oxidative stress caused by mitochondrial abnormalities play an important role in the pathophysiology of lipopolysaccharide (LPS)-induced memory impairment. Elamipretide (SS-31) is a novel mitochondrion-targeted antioxidant. However, the impact of elamipretide on the cognitive sequelae of inflammatory and oxidative stress is unknown.

Methods: We utilized MWM and contextual fear conditioning test to assess hippocampus-related learning and memory performance. Molecular biology techniques and ELISA were used to examine mitochondrial function, oxidative stress, and the inflammatory response. TUNEL and Golgi-staining was used to detect neural cell apoptosis and the density of dendritic spines in the mouse hippocampus.

Results: Mice treated with LPS exhibited mitochondrial dysfunction, oxidative stress, an inflammatory response, neural cell apoptosis, and loss of dendritic spines in the hippocampus, leading to impaired hippocampus-related learning and memory performance in the MWM and contextual fear conditioning test. Treatment with elamipretide significantly ameliorated LPS-induced learning and memory impairment during behavioral tests. Notably, elamipretide not only provided protective effects against mitochondrial dysfunction and oxidative stress but also facilitated the regulation of brain-derived neurotrophic factor (BDNF) signaling, including the reversal of important synaptic-signaling proteins and increased synaptic structural complexity.

Conclusion: These findings indicate that LPS-induced memory impairment can be attenuated by the mitochondrion-targeted antioxidant elamipretide. Consequently, elamipretide may have a therapeutic potential in preventing damage from the oxidative stress and neuroinflammation that contribute to perioperative neurocognitive disorders (PND), which makes mitochondria a potential target for treatment strategies for PND.
\end{abstract}

Keywords: Elamipretide, SS-31, Antioxidant, Mitochondrial dysfunction, Oxidative stress, Neuroinflammation, Memory impairment, Synaptic plasticity

\footnotetext{
* Correspondence: wwdd1962@aliyun.com

${ }^{1}$ Anesthesia and Operation Center, the First Medical Center, Chinese PLA General Hospital, 28th Fuxing Road, Haidian District, Beijing 100853, China Full list of author information is available at the end of the article
}

(c) The Author(s). 2019 Open Access This article is distributed under the terms of the Creative Commons Attribution 4.0 International License (http://creativecommons.org/licenses/by/4.0/), which permits unrestricted use, distribution, and reproduction in any medium, provided you give appropriate credit to the original author(s) and the source, provide a link to the Creative Commons license, and indicate if changes were made. The Creative Commons Public Domain Dedication waiver (http://creativecommons.org/publicdomain/zero/1.0/) applies to the data made available in this article, unless otherwise stated. 


\section{Introduction}

Postoperative cognitive dysfunction or decline, now defined as a type of perioperative neurocognitive disorder (PND), is one of the most common postoperative complications. It is characterized by a decline in cognitive function that occurs in patients after anesthesia and surgery in comparison with their preoperative cognitive status [1]. Surgery, especially in the elderly, causes central and peripheral oxidative stress and inflammatory responses that simultaneously affect synaptic plasticity, leading to cognitive dysfunction $[2,3]$. However, the exact pathogenetic mechanism underlying the effect of oxidative stress and neuroinflammation on cognitive function is still unclear. Our previous studies have shown that oxidative stress and inflammatory responses play an important role in the mouse memory impairment model caused by lipopolysaccharide (LPS). Alleviating the oxidative stress and inflammatory response in the brain hippocampus can improve the learning and memory function of mice [4-6]. Notably, the free radical scavenger edaravone was found to protect against surgery and LPS administration-induced memory impairment in adult rats [7].

Mitochondria are the organelles responsible for energy metabolism and have a direct impact on neuronal function and survival [8]. Mitochondria comprise the main center for energy transfer between intracellular and extracellular compartments and play important roles in the maintenance of cell function and in apoptosis [9]. However, they are also a major source of reactive oxygen species (ROS). The activation of mitochondriadependent apoptotic pathways and various inflammatory cytokines are mediated by mitochondrial ROS, which lead to the neuronal injury [10-12]. Oxidative-damage plays an important role in many clinical diseases such as ischemia-reperfusion injury $[13,14]$, neurodegenerative diseases [15-17], diabetes [18], heart failure [19], ischemic stroke [20], acute kidney injury (AKI) and chronic kidney disease (CKD) [21], age-related degenerative diseases [22], etc. Consequently, alleviating mitochondrial oxidative stress and protecting mitochondrial function provides a potential method for the treatment of these diseases.

Elamipretide (SS-31, D-Arg-Dmt-Lys-Phe- $\mathrm{NH}_{2}$ ) peptide, which was synthesized by Hazel H. Szeto and Peter W. Schiller, is a novel mitochondrial target antioxidant. Elamipretide has a dimethyltyrosine residue, allowing it to scavenge oxyradicals and inhibit linoleic acid and low-density lipoprotein oxidation [23]. This mitochondrial antioxidant peptide has the ability to eliminate ROS and increase adenosine triphosphate (ATP) in mitochondria, thus maintaining the mitochondrial membrane potential (MMP). By reducing mitochondrial ROS, elamipretide is able to prevent opening of the mitochondrial permeability transition pore (mPTP), prevent mitochondrial swelling, and reduce cytochrome c release in response to a high $\mathrm{Ca}^{2+}$ overload [24, 25]. Elamipretide is currently used as a strategy to protect against neurodegenerative diseases, inflammatory diseases, and ischemia-reperfusion injury in animal research $[16,17,19,26-29]$.

Lipopolysaccharide (LPS, endotoxin), a cell wall component of Gram-negative bacteria, is a major bacterial toll-like receptor 4 (TLR4) ligand that activates the innate immune response to infections. It has been shown to induce hippocampal oxidative stress, a neuroinflammatory response, neuronal death via apoptosis, and ultimately memory impairment, and has been widely used in preclinical models to investigate postoperative cognitive dysfunction $[4,6,30]$.

Based on the above findings, we hypothesized that during neuroinflammation, elamipretide might protect against mitochondrial dysfunction, attenuate oxidative stress and the inflammatory response in the hippocampus, and thus improve memory impairment. To test this hypothesis, we assessed the neuroprotective effects of elamipretide against LPS-induced oxidative damage and neuroinflammation in the present study. The results obtained may provide the basis for new treatment strategies for PND by making mitochondria a potential target of treatment.

\section{Materials and methods \\ Animals}

Adult male C57BL/6 mice aged 10-11 weeks and weighing 21-23 g were purchased from Vital River Laboratories Animal Technology Co. Ltd. (Beijing, China. Permit Number: SCXK (JING) 2012-0001). Mice were housed in groups of three to five per plastic cage $(24 \times 36 \times$ $24 \mathrm{~cm}$ ) under standard environmental conditions (temperature $24 \pm 1{ }^{\circ} \mathrm{C}$, a $12 \mathrm{~h}$ light-dark cycle, and $50 \% \pm 10 \%$ humidity) with ad libitum access to food and water. All experimental procedures and protocols were reviewed and approved by the Institutional Animal Care and Utilization Committee (IACUC) of the Chinese PLA General Hospital (Beijing, China) and were performed in accordance with the Guidelines for the Care and Use of Laboratory Animals of the National Institutes of Health, USA. The animals were acclimatized for 7 days before the experiment and were group-housed with the same cage mates throughout the acclimation and testing periods, and adequate measures were taken to minimize animal discomfort.

\section{Experimental protocols}

Ninety-six mice were randomly assigned to one of the following four treatment groups ( $n=24$ mice per group): a control plus placebo group (CON group), a control 
plus elamipretide group (SS-31 group), LPS plus placebo group (LPS group), and the LPS plus elamipretide group ( $\mathrm{S}+\mathrm{L}$ group).

LPS (Sigma, St. Louis, MO, USA) was dissolved in artificial cerebrospinal fluid (aCSF: $140 \mathrm{mM} \mathrm{NaCl} ; 3.0 \mathrm{mM}$ $\mathrm{KCl} ; 2.5 \mathrm{mM} \mathrm{CaCl} 2 ; 1.0 \mathrm{mM} \mathrm{MgCl}$; and $1.2 \mathrm{mM}$ $\left.\mathrm{Na}_{2} \mathrm{HPO}_{4} ; \mathrm{pH}=7.4\right)(2 \mu \mathrm{g}$ in $2 \mu \mathrm{L})$, or $2 \mu \mathrm{L}$ aCSF was stereotaxically infused into the lateral ventricle at the coordinate: $\mathrm{AP}-0.5, \mathrm{ML}+1.0$, and $\mathrm{DV}-2.0$, using a mouse brain stereotactic apparatus (Kopf Instruments, Tujunga, CA, USA) after anesthetizing the mice with ketamine/xylazine $(200 / 10 \mathrm{mg} / \mathrm{kg})$. Using a $10 \mu \mathrm{L}$ Hamilton microsyringe, the injection speed was set at $0.667 \mu \mathrm{L} / \mathrm{min}$, and the needle was held in place for 2 min following injection for proper dispersal of the drug from the tip. Mice were treated intraperitoneally with phosphate-buffered saline or elamipretide $(5 \mathrm{mg} / \mathrm{kg}$, synthesized in China by Peptides Co., Ltd., Shanghai) $30 \mathrm{~min}$ before the stereotaxic injection, and then once daily for three consecutive days thereafter. Injections $(0.2 \mathrm{~mL} / 10 \mathrm{~g}$ weight; $0.025 \%$ concentration) were alternated daily between the right and left side of the abdomen. The dose of elamipretide was chosen based on previous research showing that repeated injections of elamipretide $5 \mathrm{mg} / \mathrm{kg}$ provided maximum neuroprotective effects without any adverse effects in mouse models $[26,31]$.

\section{Behavior tests}

All assays were conducted during light-phase hours (9: 00-17:00) by observers who did not know the group of the mice until the test had been completed.

\section{Open field test}

The open field tests were performed $2 \mathrm{~h}$ before the probe tests for reference memory, according to a previous study [32]. The mice were individually placed in the apparatus consisting of four transparent Plexiglas arenas $(50 \times 50 \times 30 \mathrm{~cm})$ in which the floor and walls were covered with white paper to prevent any interference from movements of the neighboring mouse. Each mouse was released into a corner of the box and allowed to explore for $60 \mathrm{~min}$. The total grid crossing and velocity, duration, and distance in the center area were recorded during first $10 \mathrm{~min}$, which were analyzed using the ANYmaze behavioral tracking software system (Stoelting Co. Wood Dale, IL, USA). The open field apparatus was thoroughly cleaned with $5 \%$ ethyl alcohol after the test of each mouse and allowed to dry between tests.

\section{Morris water maze}

The Morris water maze (MWM) test, which is a hippocampal-dependent test of spatial learning, spatial memory, and cognitive flexibility for rodents, was performed as described previously with minor modifications $[6,32]$. The water maze was a white circular tank made of polyvinyl chloride $(118 \mathrm{~cm}$ in diameter and $42 \mathrm{~cm}$ in height) and filled with white non-toxic paint and water $\left(23 \pm 1{ }^{\circ} \mathrm{C}\right)$ to a depth of $28 \mathrm{~cm}$. The maze was placed in a room with several visual cues for orientation in the maze. The maze was divided into four quadrants, i.e., the first, second, third, and fourth quadrants. An invisible platform $(11 \mathrm{~cm}$ in diameter) was placed $1 \mathrm{~cm}$ below the water surface in the first quadrant (target quadrant). Mice were trained for the hidden platform version during acquisition, which consisted of four trials for five consecutive days. Mice were released to the MWM from all the other three quadrants, except the target quadrant, and were trained to find the hidden platform and climb onto it within $60 \mathrm{~s}$. When the mice failed to reach the platform within $60 \mathrm{~s}$, they were manually guided to the platform and allowed to stand on the platform for $15 \mathrm{~s}$ before being transferred back to cages. After that, the mouse was removed to its cage and the second animal was tested. This rotation was repeated until all animals had completed a trial. This process was repeated for subsequent trials until four trials had been completed per day for five consecutive days. After each trial, animals were towel dried and returned to their home cage under a heater for $10 \mathrm{~min}$.

The probe trial was performed on the next day after stereotactic microinjection (day 7) in the water maze without a platform. Swimming velocity, platform-site crossings, latency to the platform, and the percentage of time traveled in the different quadrants were recorded in a single 60 -s trial. Reference memory was determined by a preference for the platform area. The trajectories of the mice were recorded and analyzed using ANY-maze behavioral tracking software system (Stoelting Co. Wood Dale, IL, USA).

On days 7,8 , and 9 , working memory was tested, during which both the platform and mice were randomly placed in a novel position to assess working- or trialdependent learning and memory. In this procedure, the animal is given two trials per day. On each day, animals underwent the first training trial to ensure that all mice learned the new platform location. After $15 \mathrm{~s}$, the second trial as the test trial or matching trial was performed, in which each mouse was released from the same location as in the first trial; if it recalled the first trial, the mouse would swim a shorter path to the platform in the second trial. As the platform is moved daily, learning of platform position from the previous day cannot be transferred to the next day; hence, recall on each day during the second trial is dependent on that day's training trial and measures only temporary or working memory. Measurement of data in the second trial was conducted up to $60 \mathrm{~s}$, and the latency for mice that could not reach 
the platform in the allotted time was regarded as $60 \mathrm{~s}$. Eventually, the escape latency to the platform in the second trial was recorded as measure of temporary or working memory. The trajectories of the mice were recorded and analyzed using ANY-maze behavioral tracking software system (Stoelting Co. Wood Dale, IL, USA).

\section{Contextual fear conditioning test}

To measure the ability of hippocampus-dependent learning and memory, we employed the fear conditioning paradigm $(30 \mathrm{~cm}$ long $\times 26 \mathrm{~cm}$ wide $\times 22 \mathrm{~cm}$ high; XRXC404, Softmaze Information Technology Co. Ltd., Shanghai, China). The contextual fear conditioning test was performed as described previously with minor modifications [32]. On the acquisition day, mice were habituated for $2 \mathrm{~min}$, received a 2 -s $0.5 \mathrm{~mA}$ foot shock every $2 \mathrm{~min}$ four times, and were then returned to cages $2 \mathrm{~min}$ after the fourth shock. The day 2 trial was performed on the next day after stereotactic microinjection in the same chamber for 6 min to evaluate consolidated fear memory by analyzing the time of freezing.

\section{Tissue isolation}

As previously described by us $[4,6]$, at $6 \mathrm{~h}$ after injection of LPS, six mice in each group were decapitated. The hippocampus was rapidly removed for an enzyme-linked immunosorbent assay (ELISA). The brains of six mice sacrificed at $24 \mathrm{~h}$ in each group were quickly removed and washed in ice-cold saline. The hippocampus was dissected and separated into two halves for mitochondria isolation and immunoblotting analysis. Tissue for immunoblotting analysis was collected carefully in a sterile tube prior to being snap-frozen in liquid nitrogen and stored at $-80{ }^{\circ} \mathrm{C}$ until analysis. Transcardial perfusion was performed with ice-cold standard phosphatebuffered saline (PBS) in another six mice at $24 \mathrm{~h}$ and $72 \mathrm{~h}$ within each group. Brains were fixed in $4 \%$ paraformaldehyde for $48 \mathrm{~h}$ thereafter for terminal transferase biotinylated-dUTP nick end labeling (TUNEL) and Golgi-staining analysis, respectively. All mice were sacrificed by cervical decapitation under deep isoflurane anesthesia.

\section{Isolation of hippocampal mitochondria}

Mitochondria from the aforementioned half of the hippocampus were isolated using the tissue mitochondria isolation kit for tissue protocols (C3606, Beyotime Institute of Biotechnology, Shanghai, China). Briefly, fresh tissue samples were homogenized in ice-chilled Dounce homogenizers $(1: 10, \mathrm{w} / \mathrm{v})$ using an isolation buffer and centrifuged at $1000 \mathrm{~g}$ for $5 \mathrm{~min}$ at $4{ }^{\circ} \mathrm{C}$. Supernatants were then removed and centrifuged at $3500 \mathrm{~g}$ for $10 \mathrm{~min}$ at $4{ }^{\circ} \mathrm{C}$. The resulting sediment consisted of mitochondria. The supernatants were collected and centrifuged at $12,000 \mathrm{~g}$ for $10 \mathrm{~min}$ at $4{ }^{\circ} \mathrm{C}$ to remove sediment and to obtain cytoplasmic proteins. Appropriate amounts of mitochondrial stock solution were added to resuspend the sediment, and the protein of the mitochondria was determined by the Micro BCA protein assay kit (Beyotime Institute of Biotechnology, Shanghai, China).

\section{Determination of the mitochondrial membrane potential level}

A mitochondrial membrane potential (MMP) assay kit with $\quad 5,5^{\prime}, 6,6^{\prime}$-tetrachloro-1, $1^{\prime}, 3,3^{\prime}$ tetraethylbenzimidazolyl-carbocyanine iodide (JC-1) [C2006; Beyotime Institute of Biotechnology, Shanghai, China] was used to detect MMP. JC-1 accumulates to form J-aggregates and emits red fluorescence (Cy3, excitation/emission wave length of 525/590 nm) in mitochondria with higher membrane potentials, while JC-1 monomers emit green fluorescence (fluorescein isothiocyanate [FITC], excitation/emission wave length of 490/ $530 \mathrm{~nm}$ ) in mitochondria with lower membrane potentials. A decrease of the ratio (red: green) was thus interpreted as a decrease in MMP [33]. For this assay, different groups of $0.1 \mathrm{~mL}$ of purified mitochondria (protein concentration $0.2 \mathrm{mg} / \mathrm{mL}$ ) were incubated with $0.9 \mathrm{~mL}$ of $0.2 \mathrm{X} \mathrm{JC}-1$ staining working solution. A time scan was performed directly using a fluorescence microplate reader (Gemini EM Microplate Reader, Molecular Devices, Sunnyvale, CA, USA) with an excitation/emission wave length of $485 / 590 \mathrm{~nm}$, and observed under an Olympus BX5 fluorescence microscope imaging system (Olympus America, Melville, NY, USA).

\section{Adenosine triphosphate assay}

Adenosine triphosphate (ATP) content was measured by a firefly luciferase-based ATP assay kit (S0026, Beyotime Institute of Biotechnology, Shanghai, China) with some modifications [34]. According to the manufacturer's instructions, cold lysis buffer $(20 \mathrm{mg} / 200 \mu \mathrm{L})$ was quickly added to freshly harvested hippocampal tissue. Tissues were homogenized with a glass homogenizer on ice and centrifuged at $12,000 \mathrm{~g}$ for $5 \mathrm{~min}$ at $4{ }^{\circ} \mathrm{C}$. The detection wells had $100 \mu \mathrm{L}$ of ATP testing working solution added, were incubated for $5 \mathrm{~min}$ at room temperature, and then added to a $20 \mu \mathrm{L}$ sample or standard. The protein concentration was measured with BCA protein assay kit (Beyotime Institute of Biotechnology, Shanghai, China). Emitted light was linearly related to the ATP concentration and was measured using a microplate luminometer (GloMax ${ }^{\circ}$ 96, Promega Corporation, WI 53711, USA). Measurements were conducted in duplicate. The mean value of the duplicates was used to represent the value of each mouse. Data were normalized to the control group and expressed as a percentage of control levels. 


\section{Assessment of reactive oxygen species}

The GENMED oxidative stress reactive oxygen species (ROS) primary fluorescence assay kit for living tissue (GMS10016.4, GENMED Scientifics Inc., MA, USA) was chosen to detect ROS. 2',7'-Dichlorofluorescein diacetate (DCFH-DA) is a stain that can penetrate through the cell membrane freely. Green fluorescence is produced once DCFH-DA is oxidized by hydrogen peroxide, peroxide groups, peroxynitrite, etc. Briefly, the hippocampus was freshly harvested $24 \mathrm{~h}$ after stereotaxic injection and cold GENMED diluent $(10 \mathrm{mg} / 100 \mu \mathrm{L})$ was quickly added. Tissues was homogenized with a DOUNCE homogenizer on ice. The protein in the hippocampus was determined by the GENMED Bradford protein assay kit (GMS30030.1, GENMED Scientifics Inc., MA, USA). Fifty microliters of the supernatant was mixed with $50 \mu \mathrm{L}$ catalyst in a well of 96-well plates and incubated at room temperature for $5 \mathrm{~min}$. Subsequently, GENMED staining working solution containing DCFHDA $(100 \mu \mathrm{L})$ was added to each well and reacted for $20 \mathrm{~min}$ in the dark. Fluorescence at $490 \mathrm{~nm}$ excitation and $520 \mathrm{~nm}$ emission was read on a fluorescence microplate reader (Gemini EM Microplate Reader, Molecular Devices, Sunnyvale, CA, USA) and observed under an Olympus BX5 fluorescence microscope imaging system (Olympus America, Melville, NY, USA).

\section{Biochemical analysis Malondialdehyde}

Malondialdehyde (MDA) is a degraded oxidative lipid product from cell membranes and is used as a reliable indicator of oxidative stress [35]. The amount of MDA was measured by the reaction of one molecule of MDA with two molecules of thiobarbituric acid (TBA) to yield a pink colored chromogen. The color reaction was measured at $532 \mathrm{~nm}$ with a reference wave length of $450 \mathrm{~nm}$. The levels of MDA in the hippocampi of mice were measured using commercial assay kits (Beyotime Biotechnology Institute, Nantong, China) according to the manufacturer's instructions.

\section{Superoxide dismutase activity}

Superoxide dismutase (SOD) is an endogenous scavenger of reactive oxygen species (ROS) and is one of the major antioxidant enzymes involved in protecting the nerve tissue from oxidative stress. The activity of SOD was measured by the reaction of NBT (nitro blue tetrazolium) with two molecules of superoxide anion to yield a blue colored chromogen. SOD has the ability to inhibit the superoxide anion-free radical $\mathrm{O}_{2}{ }^{-}$. The color reaction was measured at $560 \mathrm{~nm}$ with a reference wave length of $650 \mathrm{~nm}$. The SOD activity of tissue was also measured using commercial assay kits (Beyotime
Biotechnology Institute, Nantong, China) according to the manufacturer's instructions.

\section{ELISA}

Concentrations of interleukin-6 (IL-6) and tumor necrosis factor $\alpha$ (TNF- $\alpha)$ were examined using an ELISA kit (NEOBIOSCIENCE, Beijing, China). Hippocampal tissue was homogenized in a mixture of phenylmethylsulfonyl fluoride (PMSF) and radioimmunoprecipitation assay (RIPA) lysis buffer (Solarbio Science \& Technology Co., Ltd., Beijing, China)-10 $\mu$ L PMSF: $1 \mathrm{~mL}$ RIPA lysis buffer on ice. Supernatant protein concentrations were determined after centrifugation at 12,000 rpm for $5 \mathrm{~min}$ at $4{ }^{\circ} \mathrm{C}$ with a BCA Protein Assay kit (Beyotime Institute of Biotechnology, Shanghai, China). For each sample, $5 \mu \mathrm{L}$ of extracted protein was used for detection. The procedure followed the manufacturer's instructions. The absorbance was read on a spectrophotometer at a wave length of $450 \mathrm{~nm}$. The concentrations of IL-6 and TNF$\alpha$ were calculated according to the standard curve and recorded as $\mathrm{pg} / \mathrm{mg}$ protein.

\section{Western blotting}

Hippocampal tissue was homogenized in a mixture of PMSF and RIPA lysis buffer (Solarbio Science \& Technology Co., Ltd., Beijing, China) $-10 \mu \mathrm{L}$ PMSF: $1 \mathrm{~mL}$ RIPA lysis buffer on ice. Supernatant protein concentrations were determined after centrifugation at 12,000 rpm for $5 \mathrm{~min}$ at $4{ }^{\circ} \mathrm{C}$ with a BCA Protein Assay kit (Beyotime Institute of Biotechnology, Shanghai, China). Proteins, $20 \mu \mathrm{g}$ per lane (specified in figure legends), were separated on gradient sodium dodecyl sulfate-polyacrylamide gels (SDS-PAGE) and transferred onto a polyvinylidenedifluoride (PVDF) membrane (Millipore). Blots were blocked in $5 \%$ non-fat milk or bovine serum albumin (BSA) for $2 \mathrm{~h}$ and probed with primary antibodies overnight at $4{ }^{\circ} \mathrm{C}$. The primary antibodies used in this study were rabbit anti-BDNF IgG (1:1000, catalog number: ab108319, Abcam), rabbit anti-phospho-TrkB IgG (Tyr816) (1:500, catalog number: \# ABN1381, Millipore), rabbit anti-TrkB IgG (1:1000, catalog number: \# 4603, Cell Signaling Technology), rabbit anti-postsynaptic density protein 95 (PSD-95) antibody (1:1000, catalog number: ab18258, Abcam), rabbit anti-synaptophysin (SYN) IgG (1:20000, catalog number: ab32127, Abcam), and rabbit anti-GAPDH IgG (1:10000, catalog number: ab181602, Abcam). After washing $5 \times$ in TBS-tween, horseradish peroxidase (HRP)-conjugated secondary antibodies (Cell Signaling Technology) were applied for $1 \mathrm{~h}$, rinsed again, and bands detected using ECL (Santa Cruz or Millipore). The images were digitized from the membrane and the band intensity was quantified using Gel-Pro Analyzer software, Version 3.1 or ImageJ software, version 2.0.0. 
Terminal Transferase Biotinylated-dUTP Nick end labeling The terminal transferase biotinylated-dUTP nick end labeling (TUNEL) method was performed to label cells undergoing apoptosis following the manufacturer's instructions (Roche Applied Science, Penzberg, Germany). Briefly, the brain sections were incubated in a permeabilization solution and then incubated with a TUNEL reaction mixture. Finally, the sections were incubated with $10 \mu \mathrm{g} / \mathrm{mL}$ Hoechst 33342 in a humidified dark chamber. After the TUNEL method, the sections were stained with DAPI (4,6-diamidino-2-phenylindole) and mounted with Fluoromount (SouthernBiotech, Birmingham, AL, USA), and FITC-labeled apoptotic cells were then imaged on an Olympus BX5 fluorescence microscope imaging system (Olympus America, Melville, NY, USA). The number of apoptotic neural cells per view was counted using microscopy at $\times 400$ magnification.

\section{Golgi-Cox staining}

Golgi-Cox staining was used to detect the dendritic spines of hippocampal neurons as described previously, with modification [36]. The half brain was dissected out and processed with Golgi-Cox Impregnation \& Staining System according to the manufacturer's instruction (super Golgi Kit, Bioenno Tech, LLC). After impregnation, sections (100 to $200 \mu \mathrm{m}$ ) were obtained using a vibratome, and the sections were mounted on gelatincoated glass slides and stained. Images were taken by using a Zeiss Imager II deconvolution microscope with SlideBook 5.5 software. For quantification of spines, images were acquired as a series of z-stack at $0.1 \mu \mathrm{m}$ steps to create sequential images, enabling spine counting and spine morphology measurements on 3D images using a $\times 100$ oil objective. ImageJ (Version 2.0.0-rc-69/1.52n https://imagej.nih.gov/ij/index.html) and NeuronStudio (Version 0.9.92, http://research.mssm.edu/cnic/tools-ns. html) were used to reconstruct and analyze dendritic spines, as described previously [37]. Spine densities were estimated by counting the number of spines along 100$150 \mu \mathrm{m}$ (CA1) and 50-75 $\mu \mathrm{m}$ (DG) segments of dendrites in hippocampal neurons. Three neuronal cells per brain slice, and three brain slices per animal were chosen for quantitative analysis. The number of spines was counted by double-blind hand counter.

\section{Statistical analysis}

All data were analyzed by an observer who was blind to the experimental protocol. Statistical calculations were performed using the statistical analysis software GraphPad Prism, Version 7.0 (GraphPad, San Diego, CA, USA). Data were expressed as the mean \pm standard error (SE). Intergroup comparisons were conducted by twoway analysis of variance (ANOVA) [LPS $\times$ elemipretide] followed by Tukey's post hoc test to determine significant differences between experimental groups. For acquisition training (days 1 to 5) of the MWM, data were analyzed using a two-way ANOVA (treatment $\times$ trial time) with repeated measures (trial days) followed by a Bonferroni post hoc test to analyze the difference in escape latency between each group. $P$ values $<0.05$ were considered statistically significant.

\section{Results}

General behavioral performances and anxiety responses were not changed by LPS and elamipretide treatment

Before the MWM task and contextual fear conditioning test, general behavioral performances and anxiety responses were assessed by the open field test. As shown in Fig. 1, times of grid crossings (b), velocity (c) in the whole arena, duration of stay (d), and distance of movement (e) in the center (light blue region) were recorded. Spontaneous locomotor activity, as determined by times of grid crossings and velocity in the whole arena, and exploratory behaviors in the center zone (as determined by the duration of stay and distance of movement) were comparable among all the experimental groups. No significant difference was observed in general behavioral performances and the anxiety responses after LPS and elamipretide treatment between the animals in the CON, elamipretide, LPS, and S + L groups (Fig. 1b), suggesting that the impaired performance in the LPS group was not a result of reduced locomotor ability.

Elamipretide prevented hippocampus-dependent learning and memory impairment induced by LPS

Our previous work has demonstrated that intracerebroventricular administration of LPS leads to learning and memory deficits $[4,6]$. Therefore, the protective effects of elamipretide on LPS-induced memory deficits were examined in this model. For the MWM test, all four experimental groups of mice learned to locate a hidden platform by using visual cues around the maze, as evidenced by the decreasing latencies over the training period $\left(F_{(4,218)}=29.6, P<0.001\right)$; but no difference was observed between the groups during the same day, indicating that the groups did not differ in spatial learning (Fig. 2b). On day 7 of the probe trial, the platform was removed and platform-site crossings (Fig. 2c), latency to arrive at the primary platform-site (Fig. 2d), and the amount of time the mice spent in the target quadrant where the platform was previously placed (Fig. 2e) were recorded. It was observed that elamipretide prevented spatial memory deficits caused by LPS $\left(F_{\mathrm{LPS}}=7.53\right.$, $F_{\mathrm{SS}-31}=3.7, F_{\mathrm{LPS}} \times \mathrm{SS}-31=4.31$ for platform-site crossings, $P<0.05 ; F_{\mathrm{LPS}}=12.8, F_{\mathrm{SS}-31}=4.3, F_{\mathrm{LPS}} \times \mathrm{SS}-31=5.03$ for latency to arrive at primary platform-site, $P<0.05 ; F_{\mathrm{LPS}}=$ $37.8, F_{\mathrm{SS}-31}=8.1, F_{\mathrm{LPS} \times \mathrm{SS}-31}=16.7$ and for percentage of 

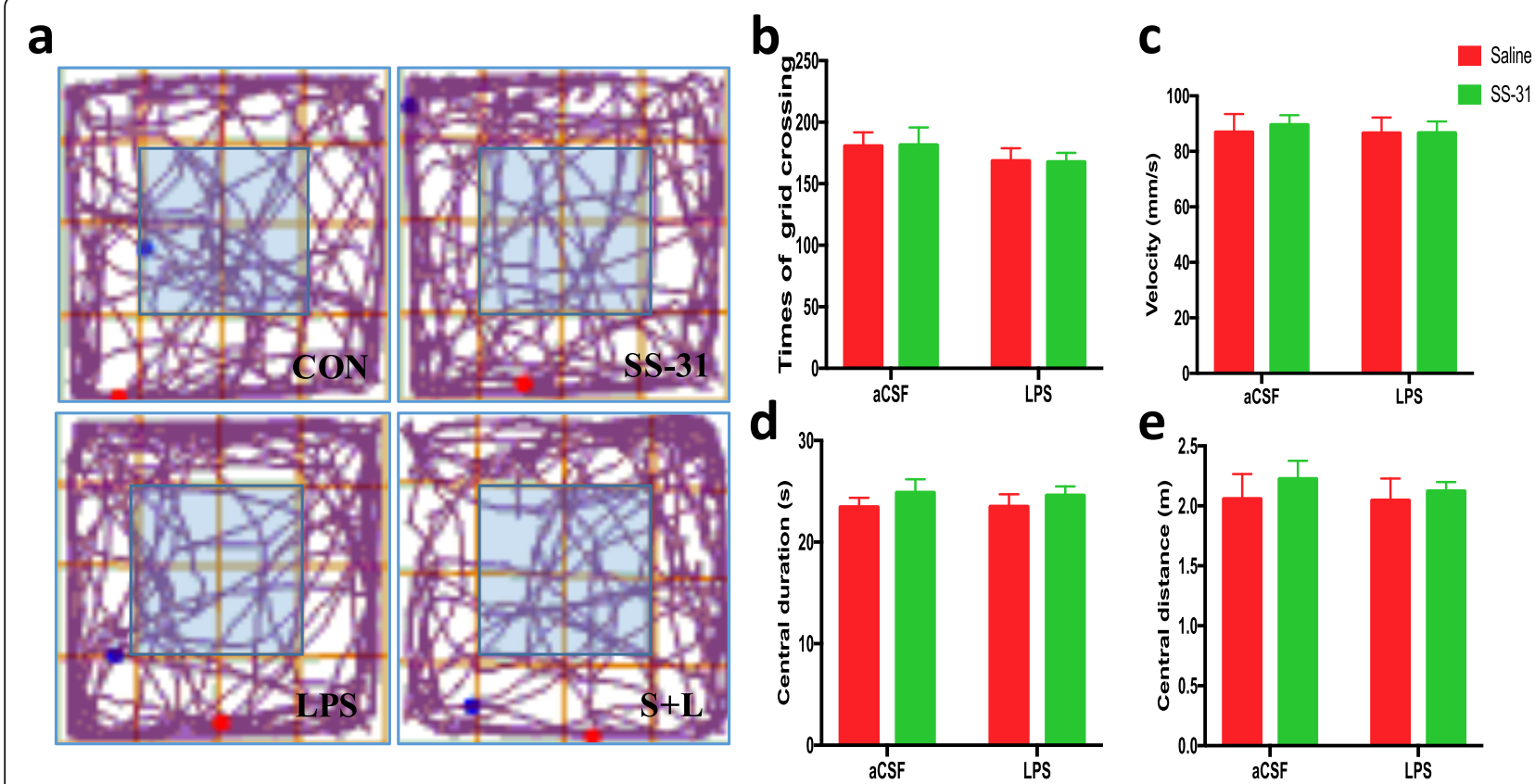

Fig. 1 Determining the effects of elamipretide (SS-31) and LPS on mice in the open field test. a Representative moving traces in the open arena during the first $10 \mathrm{~min}$. Times of grid crossings $\mathbf{b}$, velocity $\mathbf{c}$ in the whole arena, and duration of stay $\mathbf{d}$, distance of movement $\mathbf{e}$ in the center (light blue region) are expressed as the mean \pm SE $(n=10)$. SS-31 elamipretide, aCSF artificial cerebrospinal fluid, LPS lipopolysaccharide, SE standard error

time traveled in the target quadrant during the probe trial, $P<0.05$; Fig. 2c, d, e).

In the spatial working memory trial, both the platform and mice were randomly placed in a novel position to assess working- or trial-dependent learning and memory. If the animal recalls the sample trial, it will swim a shorter path to the goal on the second trial. It was observed that the escape latency needed to reach the new platform was significantly increased in the LPS group $\left(F_{\mathrm{LPS}}=11, P=0.0375\right.$; Fig. 2f) compared with the CON group. In contrast, the escape latency was returned to the CON group levels in mice treated with elamipretide.

In the contextual fear conditioning test, mice learned to express a fear response (i.e., freezing) after linking the chamber environment (CS, conditioned stimulus) with a harmful electric shock (US, unconditioned stimulus), a process called acquisition. The freezing levels among the experimental groups were similar during habituation, and increased after CS-US paired trainings (i.e., acquisition, $F_{(4,}$ 104) $=114, P<0.001$ ), so all groups of mice were able to learn associated tasks (Fig. 3b). However, the consolidated long-term fear memory, determined on the next day without US, was significantly reduced in LPS group $\left(F_{\mathrm{LPS}}=26.1\right.$, $P<0.001$; Fig. $3 \mathrm{c})$ compared with the CON group, while elamipretide significantly increased the fear memory $\left(F_{\mathrm{SS}-31}=6.63, P=0.0093\right.$; Fig. 3c) in LPS-treated mice.

Collectively, our results suggest that LPS causes hippocampus-dependent learning and memory impairment, which can be ameliorated by elamipretide treatment.

\section{Elamipretide protected the hippocampus against LPS- induced mitochondrial dysfunction by maintaining MMP and ATP levels}

We examined the effects of elamipretide on MMP and ATP levels. Our results showed that the fluorescence intensity under Cy3 (detect J-aggregates) in the LPS group was much weaker than in the CON group, which means lower membrane potentials, but elamipretide significantly increased the fluorescence intensity in the $\mathrm{S}+\mathrm{L}$ group (Fig. 4a). Consistently, the fluorescence intensity under FITC (detect JC-1 monomers) in the LPS group was much higher than in the CON group, which also means lower membrane potentials, but elamipretide significantly decreased the fluorescence intensity in the $\mathrm{S}+$ L group (Fig. 4a). The results of the fluorescence microplate reader and microplate luminometer indicated that the level of MMP ( $F_{\mathrm{LPS}}=19.6, P=0.005$; Fig. $\left.4 \mathrm{~b}\right)$ and ATP $\left(F_{\mathrm{LPS}}=25.8, P<0.001\right.$; Fig. $\left.4 \mathrm{c}\right)$ were decreased in the LPS group when compared with the CON group. Conversely, elamipretide reversed the effects on MMP $\left(F_{\mathrm{SS}-31}=9.24, P=0.04 ;\right.$ Fig. $\left.4 \mathrm{~b}\right)$ and ATP levels induced by LPS $\left(F_{\mathrm{SS}-31}=6.41, P=0.03\right.$; Fig. $\left.4 \mathrm{c}\right)$, indicating that elamipretide timely reversed LPS-induced mitochondrial dysfunction. 


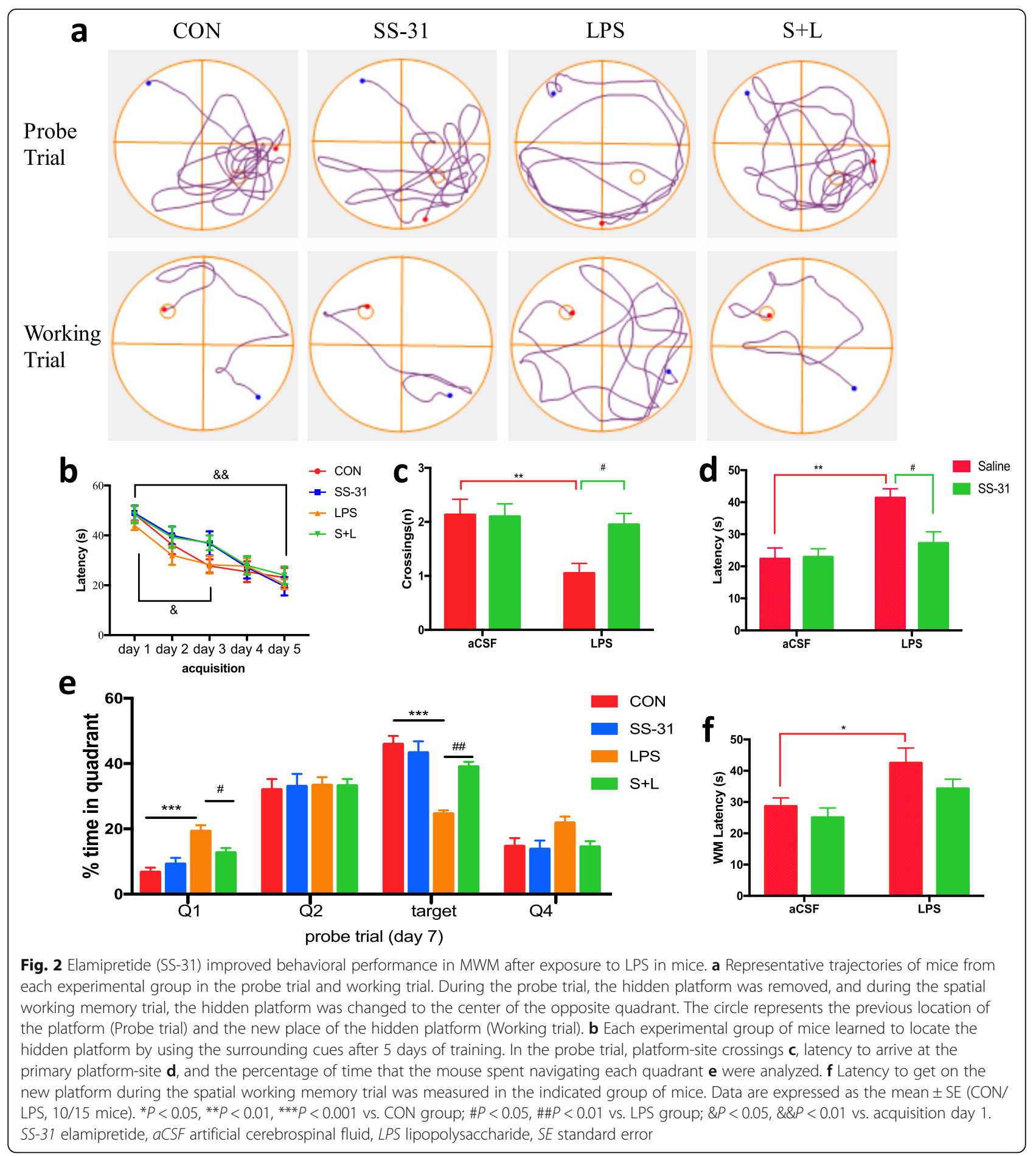

Elamipretide attenuated oxidative stress and the inflammatory response induced by LPS in the mouse hippocampus

Considering that mitochondrial dysfunction might result in oxidative stress and an inflammatory response in the LPS-treated hippocampus, we further determined the effects of elamipretide on hippocampal ROS, MDA levels,
SOD activities, and two important pro-inflammatory cytokines, TNF- $\alpha$ and IL- 6 . The fluorescence intensity of oxidized dichloro-dihydrofluorescein diacetate (DCFHDA) in the LPS group was much higher than in the CON group, which indicates increased ROS, but elamipretide significantly decreased the fluorescence intensity in the $\mathrm{S}+\mathrm{L}$ group. Compared with the CON group, LPS 


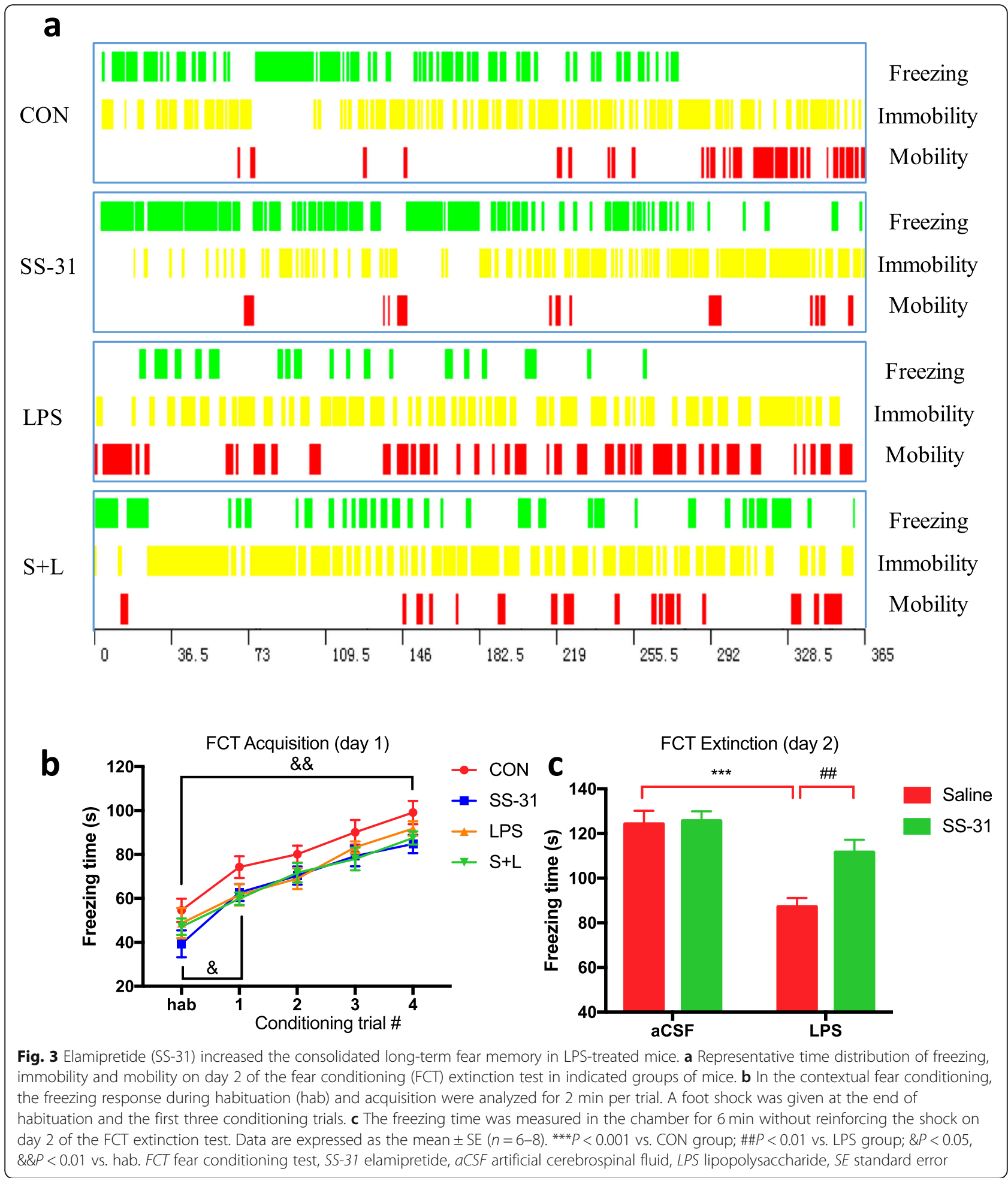

induced a significant increase in the levels of ROS $\left(F_{\mathrm{LPS}}=13.6, P=0.007\right.$; Fig. $\left.5 \mathrm{a}\right), \mathrm{MDA}\left(F_{\mathrm{LPS}}=79.6, P<\right.$ 0.001; Fig. 5b), IL-6 ( $F_{\text {LPS }}=20.1, P<0.001$; Fig. $\left.5 d\right)$, and TNF- $\alpha\left(F_{\mathrm{LPS}}=31.3, P<0.001\right.$; Fig. $\left.5 \mathrm{e}\right)$. Interestingly, elamipretide significantly attenuated abnormally increased levels of ROS $\left(F_{\mathrm{SS}-31}=5.74, P=0.05\right.$; Fig. $\left.5 \mathrm{a}\right)$, MDA $\left(F_{\mathrm{SS}-31}=7.63, P=0.003\right.$; Fig. $\left.5 \mathrm{~b}\right), \mathrm{IL}-6\left(F_{\mathrm{SS}-31}=5.83, P=\right.$ 0.018 ; Fig. $5 \mathrm{~d})$, and TNF- $\alpha\left(F_{\mathrm{SS}-31}=3.42 ; P=0.022\right.$, Fig. 5e) in the hippocampus of the $\mathrm{S}+\mathrm{L}$ group. As demonstrated in Fig. 5c, LPS induced a significant decrease 


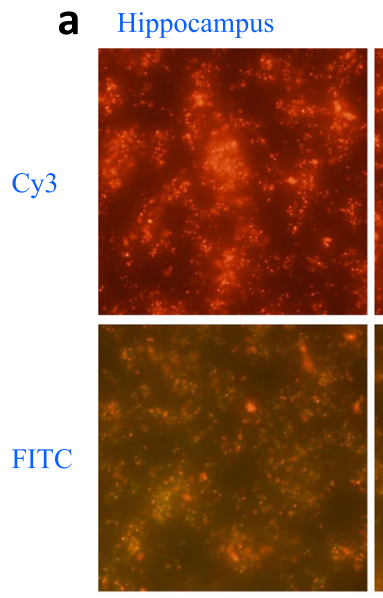

$\mathrm{CON}$
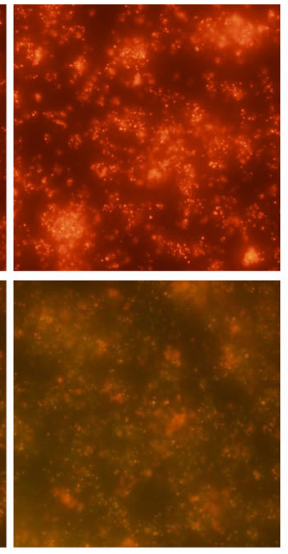

SS-31
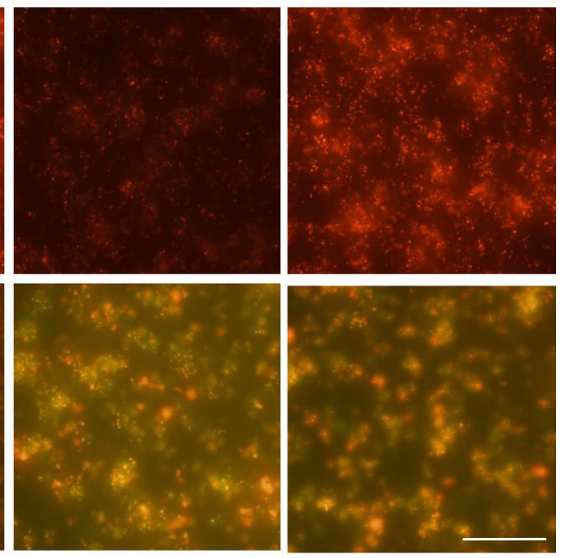

LPS

$\mathrm{S}+\mathrm{L}$
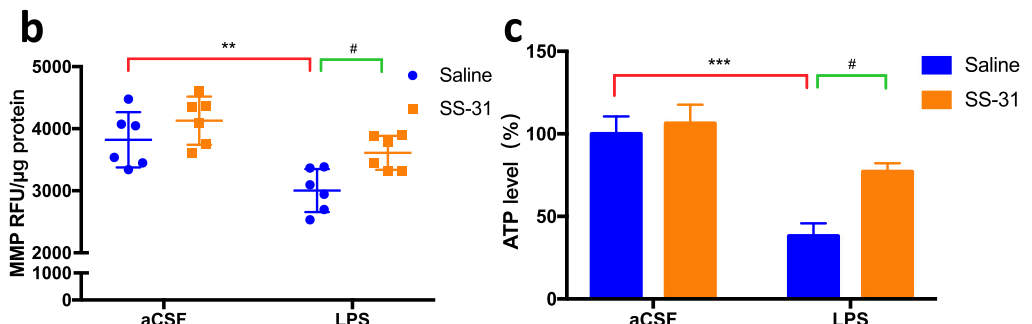

Fig. 4 Protective effects of elamipretide (SS-31) on mitochondrial dysfunction induced by LPS in the mouse hippocampus. a Representative fluorescence intensity images of J-aggregates (Cy3) and JC-1 monomers (FITC) from hippocampal mitochondria in each group. The MMP level $\mathbf{b}$ and ATP production $\mathbf{c}$ in the hippocampus were measured immediately after samples were prepared from each group. Scale bars: $20 \mu \mathrm{m}$, Data are expressed as the mean \pm SE $(n=6) .{ }^{*} P<0.01,{ }^{* *} P<0.001$ vs. CON group. $\# P<0.05$ vs. LPS group. SS-31 elamipretide, aCSF artificial cerebrospinal fluid, LPS lipopolysaccharide, SE standard error

in SOD activity $\left(F_{\mathrm{LPS}}=23, P<0.001\right)$ in the hippocampus compared with the CON group; this abnormal decrease in SOD activity was largely prevented by elamipretide $\left(F_{\mathrm{SS}-31}=3.92, P=0.006\right)$.

Our results suggested that elamipretide attenuated oxidative stress and the inflammatory response induced by LPS in the mouse hippocampus.

\section{Elamipretide significantly decreased neural cell apoptosis} in the hippocampus of LPS-treated mice

In order to observe the effect of elamipretide on LPSinduced hippocampal neural cells, we carried out a TUNEL assay. We quantified the TUNEL-positive cells using fold change. One-fold refers to the ratio of TUNEL-positive cells to the total cells in CON group. We found that LPS intraventricular injection increased TUNEL-positive cells (apoptosis) as compared with the CON group in brain hippocampal tissues of the DG region (2.62-fold versus 1fold, $P<0.001$; Fig. 6a, c) and CA1 region (1.77-fold versus 1 -fold; $P<0.001$; Fig. 6b, d). Consistent with the above findings, elamipretide significantly attenuated LPS-induced neural cell apoptosis in the hippocampal DG region (1.56fold versus 2.62-fold, $P<0.01$; Fig. $6 \mathrm{a}, \mathrm{c}$ ) and the CA1 region (1.25-fold versus 1.77-fold, $P=0.01$; Fig. $6 \mathrm{~b}, \mathrm{~d}$ ).
These results suggested that elamipretide significantly reduced neural cell apoptosis in the hippocampus of LPS-treated mice.

\section{Elamipretide enhanced the hippocampal BDNF pathway and synaptic structural complexity in mice with LPS- induced hippocampal impairment}

To assess whether the balance of brain-derived neurotrophic factor (BDNF)/TrkB was affected by LPS, the expression of $\mathrm{BDNF}, \operatorname{TrkB}$, and $\mathrm{p}$-TrkB proteins were measured using immunoblotting. As shown in Fig. 7a-e, the expression of BDNF protein was significantly decreased in the LPS-treated mice $\left(F_{\mathrm{LPS}}=67.1, P<0.001\right.$; Fig. $7 \mathrm{c})$, whereas elamipretide treatment was able to restore the BDNF protein content to a level comparable to the CON group $\left(F_{\mathrm{SS}-31}=8.66, P=0.03\right.$; Fig. $\left.7 \mathrm{c}\right)$. The total expression of TrkB protein showed no significant difference among the four groups $\left(F_{\mathrm{LPS}}<0.001, F_{\mathrm{SS}-31}=\right.$ 0.393, $P>0.05$; Fig. $7 d$ ). Further analysis of the $\mathrm{p}-\mathrm{TrkB} /$ TrkB ratio demonstrated that the $\mathrm{p}-\operatorname{TrkB} / \operatorname{TrkB}$ ratio was decreased in the LPS group compared with the CON group $\left(F_{\mathrm{LPS}}=58.4, P<0.001\right.$, Fig. $\left.7 \mathrm{e}\right)$. Elamipretide treatment significantly increased the $\mathrm{p}-\operatorname{TrkB} / \operatorname{Trk} B$ ratio 


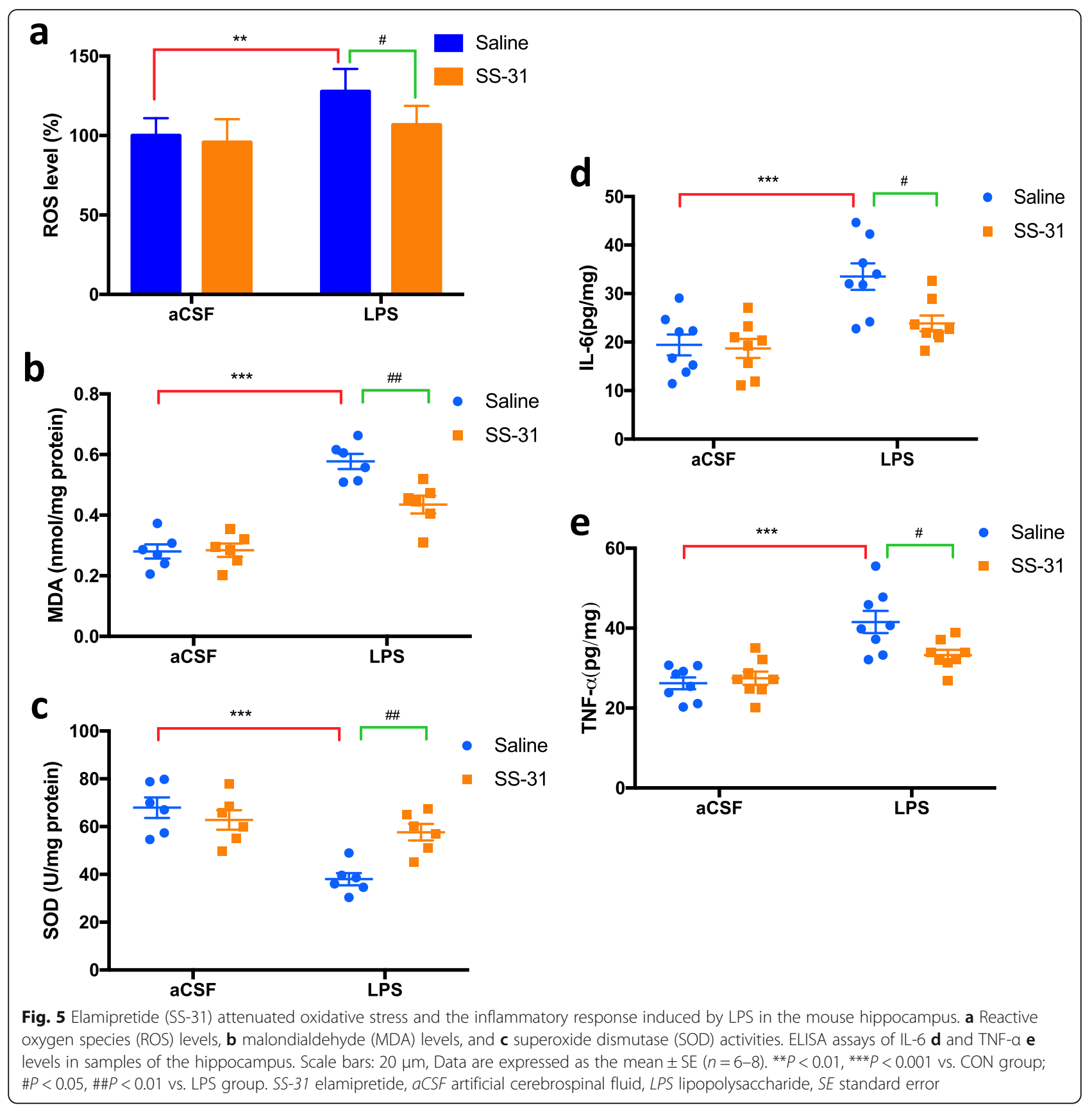

in the hippocampus of the $\mathrm{S}+\mathrm{L}$ group compared with that in the LPS group $\left(F_{\mathrm{SS}-31}=16.8, P=0.002\right.$; Fig. $\left.7 \mathrm{e}\right)$.

SYN and PSD-95 are two important regulators and indicators for determining active synaptic plasticity. The results of immunoblotting showed that SYN $\left(F_{\text {LPS }}=128, P<0.001\right.$; Fig. $7 \mathrm{~h})$ and PSD-95 $\left(F_{\mathrm{LPS}}=78.6, P<0.001\right.$; Fig. $\left.7 \mathrm{i}\right)$ were downregulated in the LPS group, but elamipretide significantly attenuated the decrease of SYN levels $\left(F_{\mathrm{SS}-31}=13.4\right.$, $P=0.002$; Fig. $7 \mathrm{~h})$ and PSD-95 levels $\left(F_{\mathrm{SS}-31}=11.5, P=0.01\right.$; Fig. 7i).

These results suggest that elamipretide enhances the hippocampal BDNF pathway and maintains the formation of active synapses in mice with LPS-induced hippocampal impairment.

\section{Elamipretide prevented the reduction of dendritic spines} on hippocampal neurons after LPS treatment

It is well established that synaptic function is closely associated with the integrity of synaptic structure. Our results have shown that elamipretide can significantly attenuate the decreased expression of synaptic proteins, including SYN and PSD-95. We further explored whether elamipretide could change the morphology of dendritic spines, where synapses are located, when 


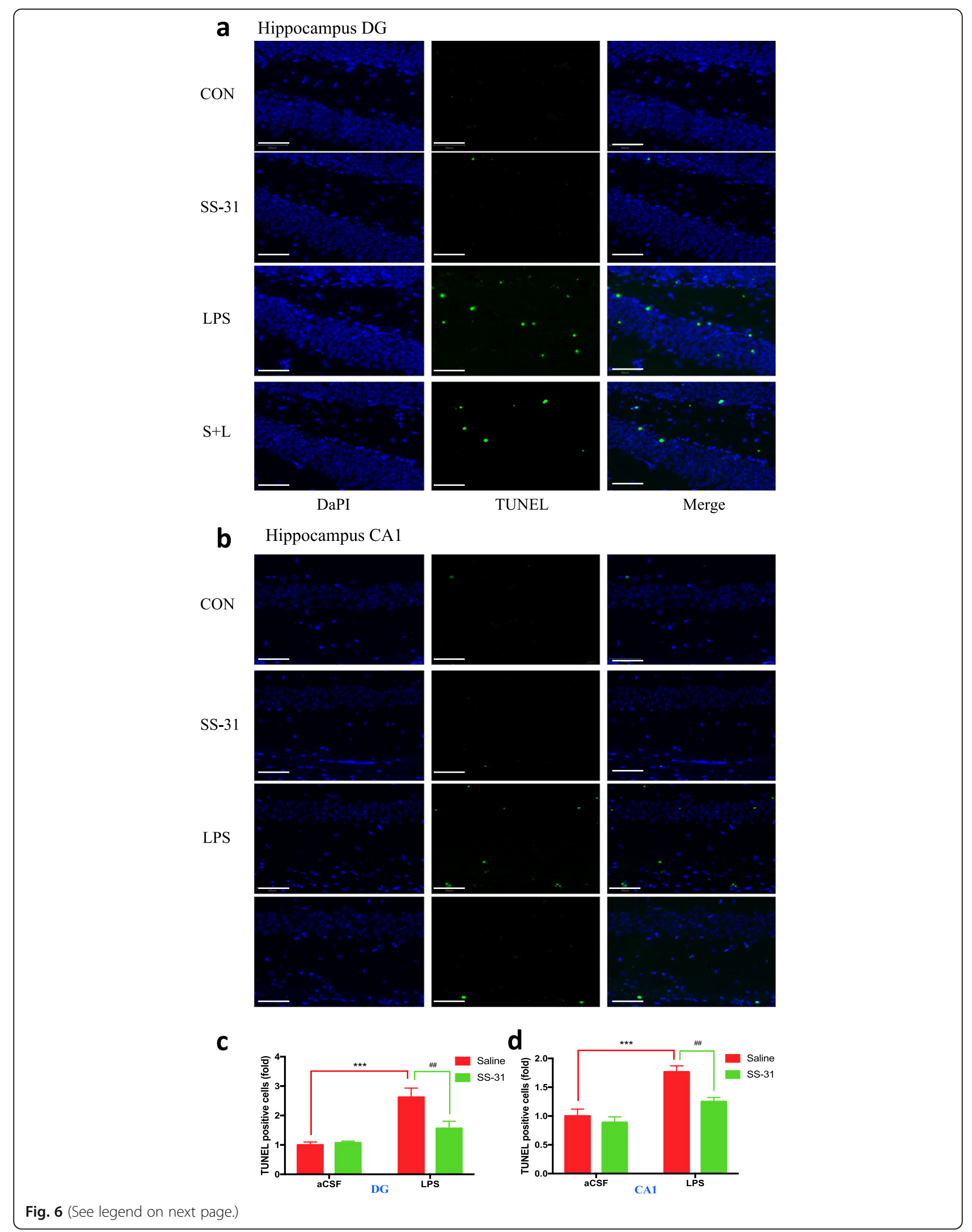


(See figure on previous page.)

Fig. 6 Effect of elamipretide (SS-31) on neural cell apoptosis in mice with hippocampal impairment. a, b TUNEL staining demonstrated neural cell apoptosis in the DG and CA1 regions of the hippocampus from indicated group of mice. $\mathbf{c}, \mathbf{d}$ The number of hippocampal apoptotic neural cells in the DG and CA1 regions of the hippocampus from indicated group of mice. Scale bars: $50 \mu \mathrm{m}$. Data are expressed as the mean $\pm S E(n=6)$. ${ }^{* * *} P<0.001$ vs. CON group; \#\#P<0.01 vs. LPS group. SS-31 elamipretide, aCSF artificial cerebrospinal fluid, LPS lipopolysaccharide, SE standard error

examined by Golgi staining. As shown in Fig. 8, the density of dendritic spines in hippocampal neurons, including granule neurons in the DG region area (green inset boxes in Fig. 8a) and pyramidal neurons in the CA1 region (red inset boxes in Fig. 8c), were significantly reduced in LPS-treated mice. In contrast, the reduced density of spines was attenuated by elamipretide treatment. When the number of dendritic spines per $10 \mu \mathrm{m}$ was counted, we found that compared with the CON group, there were fewer spines on DG neuronal dendrites $\left(F_{\mathrm{LPS}}=103, P<0.001\right.$; Fig. $\left.8 \mathrm{~b}\right)$ and CA1 neuronal dendrites $\left(F_{\mathrm{LPS}}=85.8, P<0.001\right.$; Fig. $\left.8 \mathrm{~d}\right)$ in the LPS group, whereas elamipretide significantly attenuated the decrease of dendritic spines on DG neuronal dendrites $\left(F_{\mathrm{SS}-31}=12.4, P=0.002\right.$; Fig. $\left.8 \mathrm{~b}\right)$ and CA1 neuronal dendrites $\left(F_{\mathrm{SS}-31}=9.83, P=0.03\right.$; Fig. $\left.8 \mathrm{~d}\right)$. There was no overall alteration of the morphology of spines, and distinct types of spines (mushroom spines, stubby spines, and thin spines) could be seen in the four groups.

\section{Discussion}

This study has shown that mice treated with LPS exhibited mitochondrial damage, oxidative stress response, inflammatory changes, neural cell apoptosis, and loss of dendritic spines in the hippocampus, leading to impaired hippocampus-related learning and memory performance. The behavioral tests showed that treatment with elamipretide significantly ameliorated LPS-induced hippocampus-dependent memory impairment. The protective effects of elamipretide on memory impairment in mice may be related to its mitochondria-targeted antioxidant properties, facilitating the modulation of BDNF signaling, and increasing the synaptic structural complexity in the mouse brain hippocampus.

Elamipretide is capable of successfully passing through the blood-brain barrier and targeting the inner mitochondrial membrane. It can be taken up extensively by cells and partitioned into mitochondria selectively. Intracellular concentrations of $\left[{ }^{3} \mathrm{H}\right]$-elamipretide were found to be 6-fold higher than extracellular concentrations. Studies using isolated mitochondria revealed that $\left[{ }^{3} \mathrm{H}\right]$ elamipretide is concentrated $\sim 5000$-fold in the mitochondrial pellet $[13,38]$. Thus far, a wide array of neuroprotective effects of elamipretide has been reported in different studies. Previous studies have shown that elamipretide can protect neurons from degeneration in animal models and cell lines of Alzheimer's disease (AD),
Parkinson's disease (PD), amyotrophic lateral sclerosis (ALS), multiple sclerosis (MS), and Friedreich ataxia (FA) that involve inflammatory and oxidative stress processes $[16,17,23,26,31,39]$. In view of the evidence that oxidative stress and inflammatory responses play an important role in the mouse memory impairment model caused by LPS $[4,6,30]$, our study focused on the effects of elamipretide in an LPS-induced memory impairment mouse model.

The MWM test and contextual fear conditioning were chosen as robust and reliable tests that are strongly correlated with hippocampal-dependent memory [32, 4042]. We observed that LPS-induced memory impairments were prevented by elamipretide treatment $(5 \mathrm{mg} /$ $\mathrm{kg}$ ip). The results of the current study are consistent with a previous study that showed elamipretide pretreatment shortened the escape latency in training tests and increased the crossing-platform time and target quadrant time in a probe trial with the MWM test in developing rats exposed to isoflurane [25]. In a sepsisassociated encephalopathy mouse model, elamipretide attenuated the decrease of freezing time in the contextual fear conditioning [24]. Moreover, a significant decrease was observed in isoflurane mice compared with control mice in terms of the percentage of freezing time in the contextual fear conditioning, while mice pretreated with elamipretide showed a significant increase in the percentage of freezing time compared with the isoflurane group [43]. Overall, these findings suggest a potential application of elamipretide in patients with or at risk of memory impairment.

Since elamipretide is a mitochondria-targeted protectant, we first examined whether it would improve mitochondrial function in LPS-treated mice. Mitochondria from the hippocampus was isolated using the mitochondria isolation kit for tissue protocols. As important parameters of the mitochondrial function-related index, MMP and ATP levels were measured using an assay kit. Our results showed that elamipretide protected the hippocampus against LPS-induced mitochondrial dysfunction by maintaining MMP and ATP levels. It has been proposed that LPS induces mitochondrial dysfunction in experimental models [44, 45], and the present study revealed that the brain hippocampus is one of the most vulnerable areas. Although elamipretide may target mitochondria in other tissues, our results lend support to elamipretide protecting the hippocampus from 


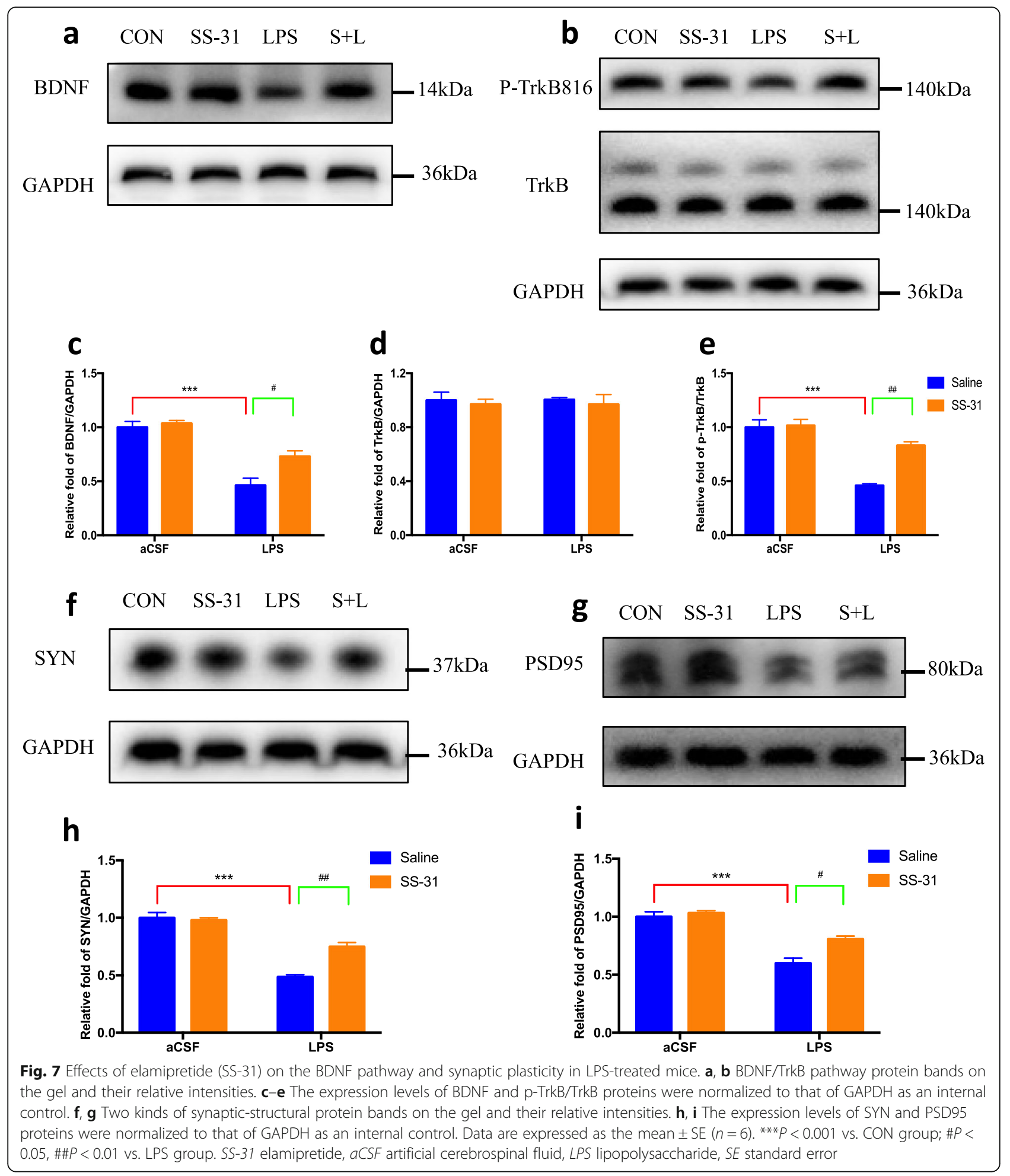

mitochondrial dysfunction in LPS-treated mice. The results are in accordance with previous reports that elamipretide protects the integrity of mitochondria by maintaining the MMP level, and protects against the mitochondrial permeability transition pore (mPTP) opening in a sepsis-associated encephalopathy mouse model [24]. Additionally, a significant decrease was observed in isoflurane mice compared with the control mice in terms of the ATP content in the hippocampus, whereas mice pretreated with elamipretide showed a 


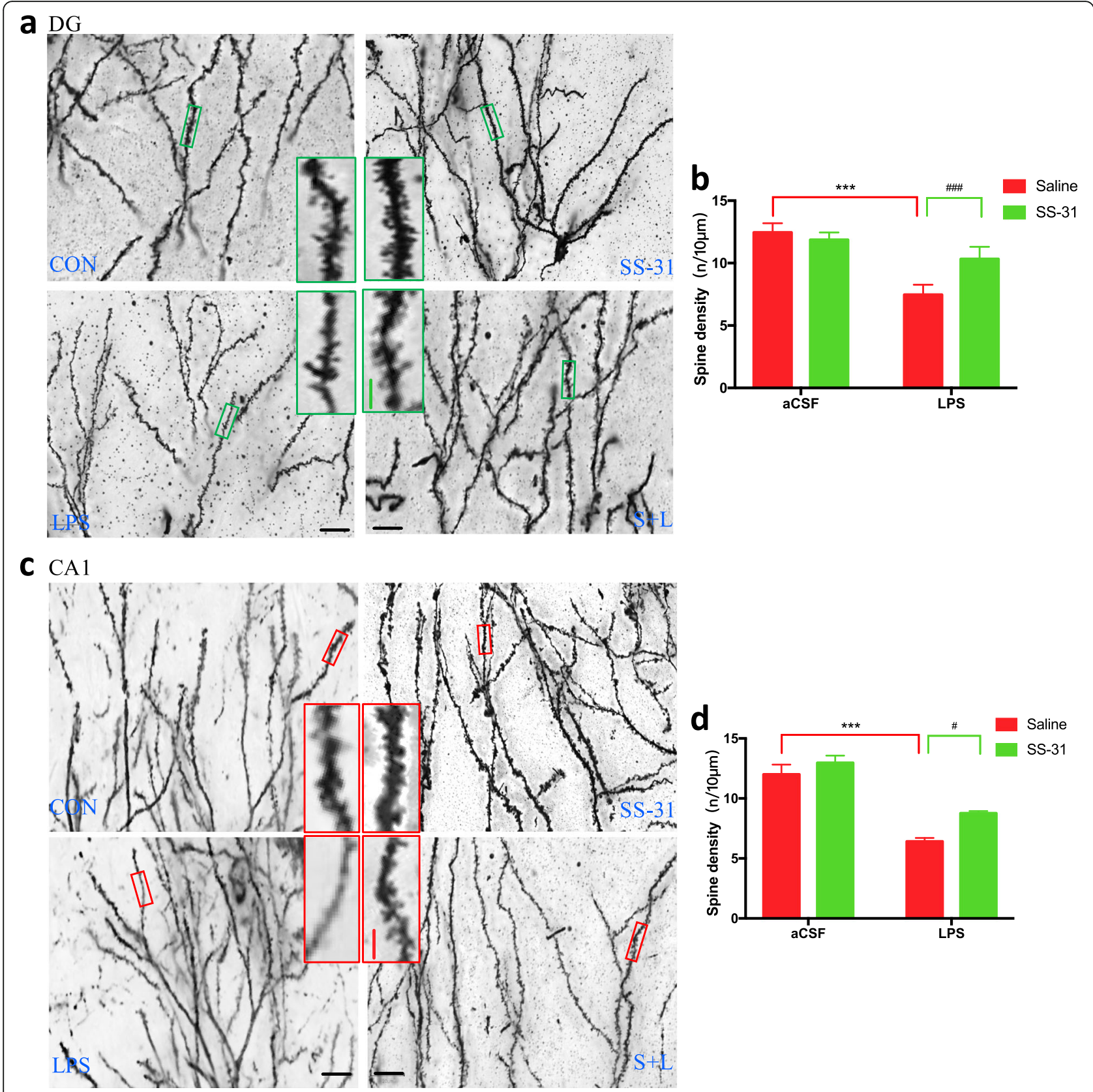

Fig. 8 Alteration of dendritic spines on hippocampal neurons visualized with Golgi staining. a, c Golgi staining of dendritic spines in hippocampal dentate granule (DG) neurons and CA1 pyramidal neurons in indicated groups of mice. A magnified view of the green and red boxed areas in a and $\mathrm{c}$ is shown on the right side of the group (CON and the LPS groups). Left side of the group: elamipretide and $S+L$ groups. b, $\mathbf{d}$ Number of dendritic spines per $10 \mu \mathrm{m}$ on hippocampal dentate granule (DG) neurons and CA1 pyramidal neurons in indicated groups of mice. Scale bars in a and c, $20 \mu \mathrm{m}$; Inset boxes, $10 \mu \mathrm{m}$. Data are expressed as the mean $\pm \mathrm{SE}(n=6)$. ${ }^{* *} P<0.001 \mathrm{vs}$. CON group; \#P<0.05, \#\#\#< 0.001 vs. LPS group. SS-31 elamipretide, aCSF artificial cerebrospinal fluid, LPS lipopolysaccharide, SE standard error

significant increase of ATP content compared with the isoflurane group [46]. Overall, treatment with elamipretide significantly improved mitochondrial function in LPS-treated mice.

Oxidative stress is defined as an imbalance between higher cellular levels of reactive oxygen species (ROS), e.g., superoxide and hydroxyl radicals, and cellular antioxidant defense $[47,48]$. If ROS are not controlled by enzymatic and non-enzymatic antioxidants, they can cause oxidative injury, i.e., peroxidation of cell membrane phospholipids, proteins (receptors and enzymes), and DNA. The brain is extremely susceptible to oxidative damage induced by ROS because it generates very high levels of ROS due to its very high aerobic 
metabolism and blood perfusion, and its relatively poor enzymatic antioxidant defense [49]. Therefore, oxidative stress participates in neuronal injury and memory impairment. Consistently, elamipretide attenuated ROS accumulation induced by LPS exposure in the present study. Decreased activity of SOD and increased levels of MDA were found after LPS microinjection in the hippocampi of animals in the LPS group. Abnormal changes in the activity of SOD and levels of MDA were partially reversed by elamipretide, suggesting that the neuroprotective effects of elamipretide might be related to its antioxidant effects. Considerable evidence implicates neuroinflammation in the pathophysiology of progressive neurodegenerative disorders $[50,51]$ and a link between increased cytokine formation and neurodegeneration has been demonstrated [52]. Several studies have demonstrated that mitochondrial reactive oxygen species (mtROS) are essential for priming the activation of NF- $\mathrm{kB}$ and the NOD-like receptor family, pyrin domain containing 3 (NLRP3) inflammasome [53, 54], which activate caspase 1 and result in processing and secretion of the pro-inflammatory cytokines. NF- $\mathrm{kB}$ is also reported to be an essential signal that promotes the expression of NLRP3 and substrates TNF- $\alpha$ and IL- 6 [53]. Previous studies have shown that LPS triggers microglia activation and consequently induces proinflammatory cytokine secretion within $6 \mathrm{~h}$ via the NF$\kappa B$ pathway in the mouse hippocampus, and that the nuclear signal of the transcription factor is strongly reduced or blocked by anti-inflammatory compounds [ 55 , 56]. Thus, it is rational that scavenging mtROS by elamipretide could suppress the increases of TNF- $\alpha$ and IL- 6 in LPS-treated mice. Elamipretide possesses the ability to block NF- $\kappa \mathrm{B}$ activation and exert a neuroprotective effect, suggesting that the neuroprotective effects of elamipretide might be related to its antineuroinflammation effects. In fact, oxidative stress and neuroinflammation exist simultaneously, and the interaction between oxygen free radicals and inflammatory factors aggravates cognitive deficiency $[57,58]$.

It is well known that LPS causes cognitive lesions and that this process involves apoptosis. As we have reported previously, LPS-induced memory impairment is associated with decreased Bcl-2 expression and increased neural cell apoptosis [6]. In this study, mitochondrial dysfunction was found after exposure to LPS, but treatment with elamipretide significantly improved mitochondrial integrity and function by maintaining MMP and ATP levels in the hippocampus of mice. Overproduction of mtROS inhibits mitochondrial electron transport, which may result in mitochondrial membrane depolarization and the initiation of apoptosis by releasing Cyt C [59]. The present study provided evidence that the mitochondrion-targeted antioxidant elamipretide significantly inhibited apoptosis and prevented memory impairment in LPS-treated mice. Thus, protection against mitochondrial dysfunction by removing mtROS will prevent the activation of the intrinsic mitochondriadependent apoptotic pathway and further reduce hippocampus-dependent memory defects in LPS-treated mice. These results indicate that mitochondria play an important role in oxidative stress and neuroinflammation induced cognitive impairment.

BDNF and its signaling pathways are firmly implicated in neuronal differentiation and survival. Numerous pieces of evidence indicate that BDNF regulates both the early and late phases of long-term potentiation in the hippocampus [60]. Its regulation of synaptic plasticity may underlie hippocampus-dependent learning and memory. BDNF exerts its neuronal protective functions through binding to the TrkB receptor. BDNF/TrkB has been shown to regulate the phosphorylation, trafficking, and expression of $N$-methyl-D-aspartate (NMDA) receptor subunits. The actions of BDNF on NMDA receptors in the hippocampus have direct implications in its ability to facilitate $\mathrm{Ca}^{2+}$ influx [43]. Studies have previously reported its role in learning, memory, and neurogenesis [61-64]. BDNF signaling through postsynaptic TrkB receptors is essential for the insertion of AMPARs (AMPA-type receptors) for postsynaptic density and the development of mature synapses [61]. Overall, BDNF/ TrkB signaling is critical for synaptic function and plasticity. The synapse-associated proteins, especially presynaptic SYN and postsynaptic PSD-95, promote synaptic plasticity [37, 65-67]. Aberrant dendrites and spines in the hippocampus are related to neurodegenerative disorders [36]. In this study, the levels of presynaptic SYN and postsynaptic PSD-95 decreased in the hippocampus of mice, accompanied by the downregulated $\mathrm{BDNF} /$ TrkB signaling after the mice were treated with LPS. Elamipretide treatment modulated and rectified BDNF/TrkB signaling and increased the levels of presynaptic SYN and postsynaptic PSD-95 in mice with hippocampal impairment. Golgi staining showed that LPS decreased dendritic spine density, but elamipretide prevented the reduction of dendritic spines on hippocampal neurons after LPS treatment in the hippocampal CA1 and DG regions. Consistently, previous studies have also reported that abnormal hippocampal neuronal plasticity was found following LPS-induced neuroinflammation $[15,68]$. This LPS-induced decrease in synaptic protein levels may contribute to impairment of synaptic plasticity and the learning and memory decline observed in behavioral tests, suggesting impairments of synaptic plasticity may be responsible for LPS-induced memory deficits. Meaningfully, in our study, treatment with elamipretide prevented the LPS-induced reduction of SYN and PSD-95 might through modulating the BDNF/TrkB 
signaling pathway. Elamipretide protected against the reduction of dendritic spines on hippocampal neurons might facilitate the protective effects of LPS-induced learning and memory impairment.

Thus, elamipretide treatment consolidated the hippocampal BDNF/TrkB signaling pathway by selectively reversing the protein expression or phosphorylation, and protected against mitochondrial dysfunction, and it specifically rescued the structure and function of the synapse against hippocampus-dependent learning and memory impairment.

\section{Conclusion}

In conclusion, our in vivo studies indicate that LPSinduced memory impairment can be attenuated by the mitochondrion-targeted antioxidant elamipretide. Its protective mechanism may be not only related to its mitochondria-targeted antioxidant activity but also the modulation of BDNF/TrkB signaling, including increasing synaptic structural complexity. Therefore, elamipretide may have a therapeutic potential in preventing the damage from oxidative stress and neuroinflammation that contributes to memory impairment, which makes mitochondria a potential target for treatment strategies for neuroinflammation-related cognitive impairment. Further investigation of the neuroprotective activity of elamipretide in the mammalian system is warranted, given the prospect that this medication will be used for prophylactic, as well as adjuvant therapy for neurodegenerative diseases resulting from oxidative and inflammatory damage.

\footnotetext{
Abbreviations

aCSF: Artificial cerebrospinal fluid; AD: Alzheimer's disease; AKI: Acute kidney injury; ALS: Amyotrophic lateral sclerosis; ANOVA: Analysis of variance; ATP: Adenosine triphosphate; BDNF: Brain-derived neurotrophic factor; BSA: Bovine serum albumin; CA1: Cornu ammonis 1; CKD: Chronic kidney disease; CS: Conditioned stimulus; DAPI: 4',6-Diamidino-2-phenylindole; DCFH-DA: Dichloro-dihydrofluorescein diacetate; DG: Dentate gyrus; dUTP: Deoxyuridine triphosphate; ELISA: Enzyme-linked immunosorbent assay; FA: Friedreich ataxia; FCT: Fear conditioning test; FITC: Fluorescein isothiocyanate; HRP: Horseradish peroxidase; IL-6: Interleukin-6; LPS: Lipopolysaccharide; MDA: Malondialdehyde; MMP: Mitochondrial membrane potential; mPTP: Mitochondrial permeability transition pore; MS: Multiple sclerosis; mtROS: Mitochondrial reactive oxygen species; MWM: Morris water maze; NBT: Nitro blue tetrazolium; NLRP3: NOD-like receptor family, pyrin domain containing 3; OFT: Open field test; PD: Parkinson's disease; PMSF: Phenylmethylsulfonyl fluoride; PND: Perioperative neurocognitive disorders; PSD-95: Postsynaptic density protein 95; PVDF: Polyvinylidene difluoride; RIPA: Radioimmunoprecipitation assay; ROS: Reactive oxygen species; SDS-PAGE: Sodium dodecyl sulphatepolyacrylamide gels; SE: Standard error; SOD: Superoxide dismutase; SS31: Szeto-Schiller-31/elamipretide/MTP-131/Bendavia/RX-31/D-Arg-Dmt-LysPhe-NH2; SYN: Synaptophysin; TBA: Thiobarbituric acid; TLR4: Toll-like receptor 4; TNF-a: Tumor necrosis factor a; TrkB: Tropomyosin receptor kinase B; TUNEL: Terminal transferase biotinylated-dUTP nick end labeling; US: Unconditioned stimulus; WB: Western blotting
}

\section{Acknowledgments}

The authors wish to thank Aisheng Hou, Meng Wu for helping animal behavioral tests, Yizheng Shi, Fuyang Cao, Ruitong Chu for helping tissue isolation, Ying Xie for helping with Golgi staining analysis, and Junhan Zhang for analysis of TUNEL staining results.

\section{Authors' contributions}

WXZ conceived the study, designed the experiments, and drafted the manuscript. ZPX, JBC, QF, YSW, and XYZ contributed to the acquisition and analysis of data. YL, XZ, and YTY contributed to the statistical analysis and manuscript editing. YFL contributed to the design of the studies, data interpretation, and manuscript editing. WDM conceived and direct the project, participated in the design and coordination, secured funding for the project, interpreted results and edited the manuscript, and takes overall responsibility for the work. All authors read and approved the final manuscript.

\section{Funding}

This research was conducted with the support of the National Natural Science Foundation of China [81671039 to WDM, 81471119 to ZPX).

Availability of data and materials

The datasets during and/or analyzed during the current study are available from the corresponding author on reasonable request.

\section{Ethics approval and consent to participate}

The animal experimentation procedures were approved by the Animal Care Committee of the Chinese People's Liberation Army General Hospital (Beijing, China). The maintenance and handling of the mice were consistent with the guidelines of the National Institutes of Health, and adequate measures were taken to minimize animal discomfort.

\section{Consent for publication}

Not applicable.

\section{Competing interests}

The authors declare that they have no competing interests.

\section{Author details}

${ }^{1}$ Anesthesia and Operation Center, the First Medical Center, Chinese PLA General Hospital, 28th Fuxing Road, Haidian District, Beijing 100853, China. ${ }^{2}$ State Key Laboratory of Toxicology Medical Countermeasures, Beijing Key Laboratories of Neuropsychopharmacology, Beijing Institute of Pharmacology and Toxicology, Academy of Military Sciences, Beijing 100850, China.

Received: 31 July 2019 Accepted: 29 October 2019

Published online: 20 November 2019

\section{References}

1. Evered L, Silbert B, Knopman DS, Scott DA, DeKosky ST, Rasmussen LS, Oh ES, Crosby G, Berger M, Eckenhoff RG, Nomenclature Consensus Working Group. Recommendations for the nomenclature of cognitive change associated with anaesthesia and surgery - 2018. Anesthesiology. 2018;129: 872-9.

2. Liu X, Wu Z, Hayashi Y, Nakanishi H. Age-dependent neuroinflammatory responses and deficits in long-term potentiation in the hippocampus during systemic inflammation. Neuroscience. 2012;216:133-42.

3. Hovens IB, Schoemaker RG, van der Zee EA, Absalom AR, Heineman E, van Leeuwen BL. Postoperative cognitive dysfunction: involvement of neuroinflammation and neuronal functioning. Brain Behav Immun. 2014;38: 202-10.

4. Zhang XY, Cao JB, Zhang LM, Li YF, Mi WD. Deferoxamine attenuates lipopolysaccharide-induced neuroinflammation and memory impairment in mice. J Neuroinflammation. 2015;12:20.

5. Wang W, Zhang L, Zhang X, Xue R, Li L, Zhao W, Fu Q Mi W, Li Y. Lentiviralmediated overexpression of the $18 \mathrm{kDa}$ translocator protein (TSPO) in the hippocampal dentate gyrus ameliorates Ips-induced cognitive impairment in mice. Front Pharmacol. 2016;7:384.

6. Zhao WX, Zhang JH, Cao JB, Wang W, Wang DX, Zhang XY, Yu J, Zhang YY, Zhang YZ, Mi WD. Acetaminophen attenuates lipopolysaccharide-induced cognitive impairment through antioxidant activity. J Neuroinflammation. 2017;14:17.

7. Wang P, Cao J, Liu N, Ma L, Zhou X, Zhang H, Wang Y. Protective effects of edaravone in adult rats with surgery and lipopolysaccharide administrationinduced cognitive function impairment. PLoS One. 2016;11:e0153708.

8. Jiang S, Nandy P, Wang W, Ma X, Hsia J, Wang C, Wang Z, Niu M, Siedlak SL, Torres $\mathrm{S}$, et al. Mfn2 ablation causes an oxidative stress response and 
eventual neuronal death in the hippocampus and cortex. Mol Neurodegener. 2018;13:5.

9. Ren R, Zhang Y, Li B, Wu Y, Li B. Effect of beta-amyloid (25-35) on mitochondrial function and expression of mitochondrial permeability transition pore proteins in rat hippocampal neurons. J Cell Biochem. 2011;112:1450-7.

10. Rigoulet M, Yoboue ED, Devin A. Mitochondrial ROS generation and its regulation: mechanisms involved in $\mathrm{H}_{2} \mathrm{O}_{2}$ signaling. Antioxid Redox Signal. 2011;14:459-68.

11. Naik E, Dixit VM. Mitochondrial reactive oxygen species drive proinflammatory cytokine production. J Exp Med. 2011;208:417-20.

12. Schon EA, Manfredi G. Neuronal degeneration and mitochondrial dysfunction. J Clin Invest. 2003;111:303-12.

13. Zhao K, Zhao GM, Wu D, Soong Y, Birk AV, Schiller PW, Szeto HH. Cellpermeable peptide antioxidants targeted to inner mitochondrial membrane inhibit mitochondrial swelling, oxidative cell death, and reperfusion injury. J Biol Chem. 2004;279:34682-90.

14. Sloan RC, Moukdar F, Frasier CR, Patel HD, Bostian PA, Lust RM, Brown DA. Mitochondrial permeability transition in the diabetic heart: contributions of thiol redox state and mitochondrial calcium to augmented reperfusion injury. J Mol Cell Cardiol. 2012;52:1009-18.

15. Sharma A, Liaw K, Sharma R, Zhang Z, Kannan S, Kannan RM. Targeting mitochondrial dysfunction and oxidative stress in activated microglia using dendrimer-based therapeutics. Theranostics. 2018:8:5529-47.

16. Zhao H, Li H, Hao S, Chen J, Wu J, Song C, Zhang M, Qiao T, Li K. Peptide SS-31 upregulates frataxin expression and improves the quality of mitochondria: implications in the treatment of Friedreich ataxia. Sci Rep. 2017:7:9840.

17. Fetisova E, Chernyak B, Korshunova G, Muntyan M, Skulachev V. Mitochondria-targeted antioxidants as a prospective therapeutic strategy for multiple sclerosis. Curr Med Chem. 2017;24:2086-114.

18. Hou Y, Li S, Wu M, Wei J, Ren Y, Du C, Wu H, Han C, Duan H, Shi Y. Mitochondria-targeted peptide SS-31 attenuates renal injury via an antioxidant effect in diabetic nephropathy. Am J Physiol Renal Physiol. 2016;310:F547-59.

19. Sabbah HN, Gupta RC, Kohli S, Wang M, Hachem S, Zhang K. Chronic therapy with elamipretide (MTP-131), a novel mitochondria-targeting peptide, improves left ventricular and mitochondrial function in dogs with advanced heart failure. Circ Heart Fail. 2016:9:e002206.

20. Kim EH, Tolhurst AT, Szeto HH, Cho SH. Targeting CD36-mediated inflammation reduces acute brain injury in transient, but not permanent, ischemic stroke. CNS Neurosci Ther. 2015;21:385-91.

21. Szeto $\mathrm{HH}$. Pharmacologic approaches to improve mitochondrial function in AKI and CKD. J Am Soc Nephrol. 2017;28:2856-65.

22. Eirin A, Li Z, Zhang X, Krier JD, Woollard JR, Zhu XY, Tang H, Herrmann SM, Lerman A, Textor SC, Lerman LO. A mitochondrial permeability transition pore inhibitor improves renal outcomes after revascularization in experimental atherosclerotic renal artery stenosis. Hypertension. 2012:60:1242-9.

23. Calkins MJ, Manczak M, Mao P, Shirendeb U, Reddy PH. Impaired mitochondrial biogenesis, defective axonal transport of mitochondria, abnormal mitochondrial dynamics and synaptic degeneration in a mouse model of Alzheimer's disease. Hum Mol Genet. 2011;20:4515-29.

24. Wu J, Zhang M, Hao S, Jia M, Ji M, Qiu L, Sun X, Yang J, Li K. Mitochondriatargeted peptide reverses mitochondrial dysfunction and cognitive deficits in sepsis-associated encephalopathy. Mol Neurobiol. 2015;52:783-91.

25. Wu J, Hao S, Sun XR, Zhang H, Li H, Zhao H, Ji MH, Yang JJ, Li K. Elamipretide (SS-31) ameliorates isoflurane-induced long-term impairments of mitochondrial morphogenesis and cognition in developing rats. Front Cell Neurosci. 2017;11:119.

26. Petri S, Kiaei M, Damiano M, Hiller A, Wille E, Manfredi G, Calingasan NY, Szeto HH, Beal MF. Cell-permeable peptide antioxidants as a novel therapeutic approach in a mouse model of amyotrophic lateral sclerosis. J Neurochem. 2006;98:1141-8.

27. Szeto HH, Liu S, Soong Y, Wu D, Darrah SF, Cheng FY, Zhao Z, Ganger M, Tow CY, Seshan SV. Mitochondria-targeted peptide accelerates ATP recovery and reduces ischemic kidney injury. J Am Soc Nephrol. 2011;22:1041-52.

28. Siegel MP, Kruse SE, Percival JM, Goh J, White CC, Hopkins HC, Kavanagh TJ, Szeto HH, Rabinovitch PS, Marcinek DJ. Mitochondrial-targeted peptide rapidly improves mitochondrial energetics and skeletal muscle performance in aged mice. Aging Cell. 2013;12:763-71.

29. Cai J, Jiang Y, Zhang M, Zhao H, Li H, Li K, Zhang X, Qiao T. Protective effects of mitochondrion-targeted peptide SS-31 against hind limb ischemia-reperfusion injury. J Physiol Biochem. 2018;74:335-43.
30. Liu Y, Zhang Y, Zheng X, Fang T, Yang X, Luo X, Guo A, Newell KA, Huang $X F$, Yu Y. Galantamine improves cognition, hippocampal inflammation, and synaptic plasticity impairments induced by lipopolysaccharide in mice. J Neuroinflammation. 2018:15:112.

31. Yang L, Zhao K, Calingasan NY, Luo G, Szeto HH, Beal MF. Mitochondria targeted peptides protect against 1-methyl-4-phenyl-1,2,3,6tetrahydropyridine neurotoxicity. Antioxid Redox Signal. 2009;11:2095-104.

32. Lu WH, Yeh NH, Huang YS. CPEB2 activates GRASP1 mRNA translation and promotes AMPA receptor surface expression, long-term potentiation, and memory. Cell Rep. 2017;21:1783-94.

33. Yu S, Zheng S, Leng J, Wang S, Zhao T, Liu J. Inhibition of mitochondrial calcium uniporter protects neurocytes from ischemia/reperfusion injury via the inhibition of excessive mitophagy. Neurosci Lett. 2016;628:24-9.

34. Bai F, Guo F, Jiang T, Wei $H$, Zhou $H$, Yin $H$, Zhong $H$, Xiong L, Wang Q. Arachidonyl-2-chloroethylamide alleviates cerebral ischemia injury through glycogen synthase kinase-3beta-mediated mitochondrial biogenesis and functional improvement. Mol Neurobiol. 2017;54:1240-53.

35. Valdes-Tovar M, Estrada-Reyes R, Solis-Chagoyan H, Argueta J, DorantesBarron AM, Quero-Chavez D, Cruz-Garduno R, Cercos MG, Trueta C, OikawaSala J, et al. Circadian modulation of neuroplasticity by melatonin: a target in the treatment of depression. Br J Pharmacol. 2018;175:3200-8.

36. Tan XJ, Dai YB, Wu WF, Kim HJ, Barros RP, Richardson TI, Yaden BC, Warner M, McKinzie DL, Krishnan V, Gustafsson JA. Reduction of dendritic spines and elevation of GABAergic signaling in the brains of mice treated with an estrogen receptor beta ligand. Proc Natl Acad Sci U S A. 2012;109:1708-12.

37. Song Y, Hu M, Zhang J, Teng ZQ, Chen C. A novel mechanism of synaptic and cognitive impairments mediated via microRNA-30b in Alzheimer's disease. EBioMedicine. 2019:39:409-21.

38. Zhao K, Luo G, Giannelli S, Szeto HH. Mitochondria-targeted peptide prevents mitochondrial depolarization and apoptosis induced by tert-butyl hydroperoxide in neuronal cell lines. Biochem Pharmacol. 2005;70:1796-806.

39. Manczak M, Mao P, Calkins MJ, Cornea A, Reddy AP, Murphy MP, Szeto HH, Park B, Reddy PH. Mitochondria-targeted antioxidants protect against amyloid-beta toxicity in Alzheimer's disease neurons. J Alzheimers Dis. 2010; 20(Suppl 2):S609-31.

40. Tucker LB, Velosky AG, McCabe JT. Applications of the Morris water maze in translational traumatic brain injury research. Neurosci Biobehav Rev. 2018;88: 187-200.

41. D'Hooge R, De Deyn PP. Applications of the Morris water maze in the study of learning and memory. Brain Res Brain Res Rev. 2001;36:60-90.

42. Morris R. Developments of a water-maze procedure for studying spatial learning in the rat. J Neurosci Methods. 1984;11:47-60.

43. Wu J, Zhang M, Li H, Sun X, Hao S, Ji M, Yang J, Li K. BDNF pathway is involved in the protective effects of SS-31 on isoflurane-induced cognitive deficits in aging mice. Behav Brain Res. 2016;305:115-21.

44. Ren JD, Wu XB, Jiang R, Hao DP, Liu Y. Molecular hydrogen inhibits lipopolysaccharide-triggered NLRP3 inflammasome activation in macrophages by targeting the mitochondrial reactive oxygen species. Biochim Biophys Acta. 1863;2016:50-5.

45. Kozlov AV, Duvigneau JC, Miller I, Nurnberger S, Gesslbauer B, Kungl A, Ohlinger W, Hartl RT, Gille L, Staniek K, et al. Endotoxin causes functional endoplasmic reticulum failure, possibly mediated by mitochondria. Biochim Biophys Acta. 2009;1792:521-30.

46. Wu J, Li H, Sun X, Zhang H, Hao S, Ji M, Yang J, Li K. A mitochondriontargeted antioxidant ameliorates isoflurane-induced cognitive deficits in aging mice. PLoS One. 2015;10:e0138256.

47. Khan MS, Ali T, Kim MW, Jo MH, Jo MG, Badshah H, Kim MO. Anthocyanins protect against LPS-induced oxidative stress-mediated neuroinflammation and neurodegeneration in the adult mouse cortex. Neurochem Int. 2016;100:1-10.

48. Yatin SM, Varadarajan S, Butterfield DA. Vitamin E prevents Alzheimer's amyloid beta-peptide (1-42)-induced neuronal protein oxidation and reactive oxygen species production. J Alzheimers Dis. 2000;2:123-31.

49. Rimessi A, Previati M, Nigro F, Wieckowski MR, Pinton P. Mitochondrial reactive oxygen species and inflammation: molecular mechanisms, diseases and promising therapies. Int J Biochem Cell Biol. 2016;81:281-93.

50. Gao HM, Hong JS. Why neurodegenerative diseases are progressive: uncontrolled inflammation drives disease progression. Trends Immunol. 2008:29.357-65

51. Infante-Duarte C, Waiczies S, Wuerfel J, Zipp F. New developments in understanding and treating neuroinflammation. J Mol Med (Berl). 2008;86: 975-85. 
52. da Silva DJ, Borges AF, Souza PO, de Souza PR, Cardoso CR, Dorta ML, de Oliveira MA, Teixeira AL, Ribeiro-Dias F. Decreased toll-like receptor 2 and toll-like receptor 7/8-induced cytokines in Parkinson's disease patients. Neuroimmunomodulation. 2016:23:58-66.

53. Bauernfeind FG, Horvath G, Stutz A, Alnemri ES, MacDonald K, Speert D, Fernandes-Alnemri T, Wu J, Monks BG, Fitzgerald KA, et al. Cutting edge: NF-kappaB activating pattern recognition and cytokine receptors license NLRP3 inflammasome activation by regulating NLRP3 expression. J Immunol. 2009;183:787-91.

54. Zhou R, Yazdi AS, Menu P, Tschopp J. A role for mitochondria in NLRP3 inflammasome activation. Nature. 2011:469:221-5.

55. McGeer PL, McGeer EG. Inflammation of the brain in Alzheimer's disease: implications for therapy. J Leukoc Biol. 1999;65:409-15.

56. McGeer PL. Cyclo-oxygenase-2 inhibitors: rationale and therapeutic potential for Alzheimer's disease. Drugs Aging. 2000;17:1-11.

57. Ullah F, Ali T, Ullah N, Kim MO. Caffeine prevents D-galactose-induced cognitive deficits, oxidative stress, neuroinflammation and neurodegeneration in the adult rat brain. Neurochem Int. 2015;90:114-24.

58. Godbout JP, Chen J, Abraham J, Richwine AF, Berg BM, Kelley KW, Johnson RW. Exaggerated neuroinflammation and sickness behavior in aged mice following activation of the peripheral innate immune system. FASEB J. 2005; 19:1329-31.

59. Fleury C, Mignotte B, Vayssiere JL. Mitochondrial reactive oxygen species in cell death signaling. Biochimie. 2002;84:131-41.

60. Leal G, Afonso PM, Salazar IL, Duarte CB. Regulation of hippocampal synaptic plasticity by BDNF. Brain Res. 2015;1621:82-101.

61. Chakravarthy S, Saiepour MH, Bence M, Perry S, Hartman R, Couey JJ, Mansvelder HD, Levelt CN. Postsynaptic TrkB signaling has distinct roles in spine maintenance in adult visual cortex and hippocampus. Proc Natl Acad Sci U S A. 2006;103:1071-6.

62. Fan $D$, Li J, Zheng B, Hua L, Zuo Z. Enriched environment attenuates surgery-induced impairment of learning, memory, and neurogenesis possibly by preserving BDNF expression. Mol Neurobiol. 2016;53:344-54.

63. Kang SS, Zhang Z, Liu X, Manfredsson FP, Benskey MJ, Cao X, Xu J, Sun YE, Ye K. TrkB neurotrophic activities are blocked by alpha-synuclein, triggering dopaminergic cell death in Parkinson's disease. Proc Natl Acad Sci U S A. 2017;114:10773-8

64. Yin C, Deng Y, Liu Y, Gao J, Yan L, Gong Q. Icariside II ameliorates cognitive impairments induced by chronic cerebral hypoperfusion by inhibiting the amyloidogenic pathway: involvement of BDNF/TrkB/CREB signaling and upregulation of PPARalpha and PPARgamma in rats. Front Pharmacol. 2018;9:1211.

65. Zhang J, Dong Y, Zhou C, Zhang Y, Xie Z. Anesthetic sevoflurane reduces levels of hippocalcin and postsynaptic density protein 95 . Mol Neurobiol. 2015:51:853-63.

66. Lu H, Liufu N, Dong Y, Xu G, Zhang Y, Shu L, Soriano SG, Zheng H, Yu B, Xie $Z$. Sevoflurane acts on ubiquitination-proteasome pathway to reduce postsynaptic density 95 protein levels in young mice. Anesthesiology. 2017; 127:961-75.

67. Liu A, Zhang Y, Han L, He G, Xie W, Zhou Z, Jia Z. Regulation of neurotransmitter release by amyloid precursor protein through synapsin phosphorylation. Neurochem Res. 2019;44:683-91.

68. Tweedie D, Ferguson RA, Fishman K, Frankola KA, Van Praag H, Holloway HW Luo W, Li Y, Caracciolo L, Russo I, et al. Tumor necrosis factor-alpha synthesis inhibitor 3,6'-dithiothalidomide attenuates markers of inflammation, Alzheimer pathology and behavioral deficits in animal models of neuroinflammation and Alzheimer's disease. J Neuroinflammation. 2012;9:106.

\section{Publisher's Note}

Springer Nature remains neutral with regard to jurisdictional claims in published maps and institutional affiliations.

Ready to submit your research? Choose BMC and benefit from:

- fast, convenient online submission

- thorough peer review by experienced researchers in your field

- rapid publication on acceptance

- support for research data, including large and complex data types

- gold Open Access which fosters wider collaboration and increased citations

- maximum visibility for your research: over $100 \mathrm{M}$ website views per year

At BMC, research is always in progress.

Learn more biomedcentral.com/submissions 